\title{
Faint AGNs at $z>4$ in the CANDELS GOODS-S field: looking for contributors to the reionization of the Universe ${ }^{\star}$
}

\author{
E. Giallongo ${ }^{1}$, A. Grazian ${ }^{1}$, F. Fiore ${ }^{1}$, A. Fontana ${ }^{1}$, L. Pentericci ${ }^{1}$, E. Vanzella ${ }^{2}$, M. Dickinson ${ }^{3}$, D. Kocevski ${ }^{4}$, \\ M. Castellano ${ }^{1}$, S. Cristiani ${ }^{5}$, H. Ferguson ${ }^{6}$, S. Finkelstein ${ }^{7}$, N. Grogin ${ }^{6}$, N. Hathi ${ }^{8}$, A. M. Koekemoer ${ }^{6}$, \\ J. A. Newman ${ }^{9}$, and M. Salvato ${ }^{10}$
}

1 INAF-Osservatorio Astronomico di Roma, via Frascati 33, 00040 Monteporzio, Italy e-mail: emanuele.giallongo@oa-roma.inaf.it

2 INAF-Osservatorio Astronomico di Bologna, via Ranzani 1, 40127 Bologna, Italy

NOAO, 950 N. Cherry Avenue, Tucson, AZ 85719, USA

4 Department of Physics and Astronomy, University of Kentucky, Lexington, KY 40506, USA

5 INAF-Osservatorio Astronomico di Trieste, via G.B. Tiepolo 11, 34131 Trieste, Italy

${ }^{6}$ Space Telescope Science Institute, 3700 San Martin Drive, Baltimore, MD 21218, USA

7 Department of Astronomy, The University of Texas at Austin, Austin, TX 78712, USA

8 Aix-Marseille Université, CNRS, LAM (Laboratoire d'Astrophysique de Marseille) UMR 7326, 13388 Marseille, France

9 Department of Physics and Astronomy, University of Pittsburgh, 3941 O'Hara Street, Pittsburgh, PA 15260, USA

10 Max Planck Institute for Extraterrestrial Physics, Giessenbachstrasse 1, 85748 Garching bei Munchen, Germany

Received 13 November 2014 / Accepted 10 February 2015

\section{ABSTRACT}

Context. Establishing the number of faint active galactic nuclei (AGNs) at $z=4-6$ is crucial to understanding their cosmological importance as main contributors to the reionization of the Universe.

Aims. In order to derive the AGN contribution to the cosmological ionizing emissivity we have selected faint AGN candidates at $z>4$ in the CANDELS GOODS-South field, which is one of the deepest fields with extensive multiwavelength coverage from Chandra, HST, Spitzer, and various ground-based telescopes.

Methods. We have adopted a relatively novel criterion. As a first step, high redshift galaxies are selected in the NIR $H$ band down to very faint levels $(H \leq 27)$ using reliable photometric redshifts. At $z>4$ this corresponds to a selection criterion based on the galaxy rest-frame UV flux. AGN candidates are then picked up from this parent sample if they show X-ray fluxes above a threshold of $F_{\mathrm{X}} \sim 1.5 \times 10^{-17} \mathrm{erg} \mathrm{cm}^{-2} \mathrm{~s}^{-1}(0.5-2 \mathrm{keV})$, corresponding to a probability of spurious detections of $2 \times 10^{-4}$ in the deep X-ray 4 Ms Chandra image.

Results. We have found $22 \mathrm{AGN}$ candidates at $z>4$ and we have derived the first estimate of the UV luminosity function in the redshift interval $4<z<6.5$ and absolute magnitude interval $-22.5 \lesssim M_{1450} \lesssim-18.5$ typical of local Seyfert galaxies. The faint end of the derived luminosity function is about two to four magnitudes fainter at $z \sim 4-6$ than that derived from previous UV surveys. We estimated ionizing emissivities and hydrogen photoionization rates in the same redshift interval under reasonable assumptions and after discussion of possible caveats, the most important being the large uncertainties involved in the estimate of photometric redshift for sources with featureless, almost power-law SEDs and/or low average escape fraction of ionizing photons from the AGN host galaxies. Both effects could, in principle, significantly reduce the estimated average volume densities and/or ionizing emissivities, especially at the highest redshifts.

Conclusions. At $z=4-6.5$ we argue that, under reasonable evaluations of possible biases, the probed AGN population can produce photoionization rates consistent with that required to keep the intergalactic medium observed in the Lyman- $\alpha$ forest of high redshift QSO spectra highly ionized, providing an important contribution to the cosmic reionization.

Key words. quasars: general - dark ages, reionization, first stars

\section{Introduction}

The process of cosmic reionization remains one of the most important and puzzling problems for cosmology. The reionization of the Universe, after its neutral phase followed by recombination, appears completed at $z \sim 6$ as inferred from the high ionization level of the intergalactic medium (IGM) observed in the Lyman- $\alpha$ forest of high redshift QSO spectra (Fan et al. 2006). The epoch when the process becomes important is still unknown, although some hints come from the level of Thompson scattering

\footnotetext{
* Appendices are available in electronic form at http://www. aanda.org
}

of the cosmic microwave background (CMB) radiation observed by Wilkinson Microwave Anisotropy Probe (WMAP) and Planck and by the analysis of the kinematic Sunayev-Zeldovich effect (Hinshaw et al. 2013; George et al. 2015). The common wisdom is that in the epoch corresponding to the redshift interval $z \sim 6-10$ the Universe quickly changed its thermal and ionization state. However, the identification of the source populations emitting enough UV radiation to reionize the Universe is still an open issue.

Over more than fifteen years extensive studies have been performed to identify the ionizing population among the high redshift star forming galaxies or the bright active galactic nucleus 
(AGN) population. Since the apparent number density of bright QSOs and AGNs rapidly decreases at $z>3$ (e.g. Masters et al. 2012) it is usually assumed that QSOs do not produce enough ionizing emissivity at $z>4$. Estimates based on simple parametric extrapolations both in luminosity and redshifts of the AGN luminosity function imply values for the cosmological emissivity of $10^{23} \mathrm{erg} \mathrm{Mpc}^{-3} \mathrm{~Hz}^{-1} \mathrm{~s}^{-1}$ (e.g. Haardt \& Madau 2012). This is one order of magnitude smaller than that required to keep the intergalactic medium highly ionized at $z \sim 6$, even though each bright QSO is able to ionize its neighbourhood up to a distance of approximately $10 \mathrm{Mpc}$ at $z \sim 5.5$ (e.g. Prochaska et al. 2009; Songaila \& Cowie 2010; Worseck et al. 2014).

As a consequence, the more common star forming galaxies are thought to be responsible for the cosmic reionization. Their contribution depends on their abundance at low luminosities and on the average fraction of ionizing UV flux escaping from each galaxy into its neighbourhood. At very high redshifts $(z>5)$, direct measures of the ionizing Lyman continuum flux from galaxies are not viable owing to the saturated hydrogen absorption by the IGM observed in QSO spectra. Estimates at lower redshifts $(z \sim 3-4)$ become feasible, but the values rely on specific assumptions and appear discrepant. For example, recent analyses suggest average ionizing escape fractions of $\sim 10-15 \%$ (Nestor et al. 2011; Mostardi et al. 2013), but their $z \sim 3$ candidates show ionizing continua often slightly shifted in position with respect to the non-ionizing flux, which suggests significant contamination by foreground low redshift interlopers (Vanzella et al. 2012a). Other estimates based on spectroscopic and very deep broad- or narrowband imaging gave only upper limits of $<5 \%$ (Vanzella et al. 2010; Boutsia et al. 2011). At present the results on the estimated ionizing escape fraction for this relatively bright star forming population at $z \sim 3$ are not conclusive.

The faintest dwarf high redshift galaxies could behave in a different way showing larger escape fractions (see e.g. Fontanot et al. 2014) and could be significant contributors to the cosmic reionization (Ferrara \& Loeb 2013; Wise et al. 2014; Yue et al. 2014). However, some assumptions on the abundance at $z \sim 7-10$ of the faintest galaxies down to $M_{U V} \sim-13$ and on their escape fractions $(\sim 15-80 \%)$ are needed to make the star forming galaxy population a major contributor to the reionization (e.g. Finkelstein et al. 2012; Robertson et al. 2013). A recent evaluation by Finkelstein et al. (2014) indicates escape fractions of $\sim 13 \%$ to keep the IGM ionized up to $z \sim 6$ and mildly ionized to $z \sim 8$, although uncertainties are very large. Specific searches are starting in lensing galaxy clusters to measure the escape of Lyman continuum flux in intrinsically faint galaxies which are gravitationally lensed and amplified in luminosity by the intervening cluster (see e.g. Vanzella et al. 2012b; Ishigaki et al. 2015).

All these uncertainties in the galaxy contribution leave open the possibility that AGNs could still play a leading role in contributing to the cosmological ionizing background. The estimate of their contribution, however, is mainly affected by the poor knowledge of the faint end slope of their luminosity function, especially at high redshifts, owing to the lack of deep AGN surveys at various wavelengths (Shankar \& Mathur 2007).

The traditional view based on an evolutionary ionizing QSO background increasing from the local value out to $z=2-3$ and then quickly decreasing (see e.g Haardt \& Madau 2012) is changing thanks to the recent multiwavelength deep surveys at $z>3$ (Glikman et al. 2011; Civano et al. 2011; Fiore et al. 2012), which show the presence of a considerable number of previously unknown faint AGNs able to produce a steep luminosity function. The presence of this faint population is changing our estimate of the AGN contribution to the ionizing UV background, although the selection of faint AGNs at the highest redshifts $z \sim 6$ is difficult with the current instrumentation. More importantly, the inclusion of X-ray detection in the selection methods enables us to extend the knowledge of the luminosity function to even fainter X-ray limits (Civano et al. 2011; Fiore et al. 2012) of the order of $L(2-10 \mathrm{KeV})>10^{43} \mathrm{erg} \mathrm{s}^{-1}$ at $z>4$. In particular, the novel technique proposed by Fiore et al. (2012) relies on the selection of faint high redshift AGNs among the high- $z$ galaxies selected in NIR images (e.g. $H$ band) that show any detection in deep and high resolution Chandra X-ray images.

A first estimate of the volume density of faint AGNs could also have important implications concerning the abundance and mass of the supermassive black hole seeds (Volonteri 2012) and their early growth (Volonteri \& Silk 2014). Important constraints are also expected for the initial spectrum of the density perturbations related to the nature, cold or warm, of dark matter (see e.g. Menci et al. 2013).

In Giallongo et al. (2012) we made a first attempt to estimate the possible contribution to the cosmic reionization by faint high- $z$ AGNs based on the results provided by deep X-ray surveys and on a joint galaxy-AGN model prediction. We found that faint AGNs might be able to reionize the high redshift IGM provided that their average volume density only gradually declined from $z \sim 3$ to $z \sim 6.5$ as predicted by the model.

In the present paper we have produced a sample of galaxies selected in the Candels/GOODS-S/ChandraDeepField-South field using one of the deepest NIR, optical, and X-ray databases, and we have built a sample of AGN candidates based on X-ray detection in the same $H$ band galaxy position, following the recipe in Fiore et al. (2012). In Sect. 2 we describe the CANDELS photometric parent catalogue of high redshift galaxies. In Sect 3. we describe the AGN selection by means of the Chandra $4 \mathrm{Ms}$ image. In Sect. 4 we present an estimate of the faint end AGN UV luminosity function. In Sect. 5 we show predictions about the AGN ionizing emissivity and photoionization rate, and finally in Sect. 6 we discuss some possible caveats. Throughout the paper we adopt round cosmological parameters $\Omega_{\Lambda}=0.7, \Omega_{0}=0.3$, and Hubble constant $h=0.7$ in units of $100 \mathrm{~km} \mathrm{~s}^{-1} \mathrm{Mpc}^{-1}$. Apparent magnitudes are in the AB photometric system.

\section{The CANDELS GOODS-S catalogue}

The CANDELS area (Grogin et al. 2011; Koekemoer et al. 2011) with deep Chandra coverage is providing the best dataset in terms of deep NIR photometry and relatively wide area. The NIR images obtained with the Wide Field Camera 3 (WFC3) are particularly important in terms of angular resolution and depth, sampling the AGN UV emission $(<3000 \AA)$ at $z>4$. Among the various CANDELS fields, in this preliminary analysis we have selected the GOODS-South field where the deepest $4 \mathrm{Ms}$ Chandra images are ideal for the selection of high redshift AGNs. The field covers an area of $\sim 170 \mathrm{sq}$. arcmin at a mean depth of $H=27.5$. The dataset also includes very deep imaging by the IRAC instrument on board the Spitzer Space Telescope coming from the Spitzer Extended Deep Survey (SEDS; Ashby et al. 2013) and covering the CANDELS fields to 26 AB mag $(3 \sigma)$ at both 3.6 and $4.5 \mu \mathrm{m}$. The Spitzer infrared bands sample the rest-frame optical region of AGNs at $z>4$. We adopted the official photometric catalogue released by Guo et al. (2013) selecting galaxies in the WFC3 $H$ band. The total number of 
sources detected in the GOODS-South fields at a mean depth of $H=27.5$ is about 28500 .

Because of the complexity of the GOODS-South exposure map in the $H$ band, the field is composed of several sub-areas with the associated magnitude limits. Simulations have been performed to assess the detection completeness as described in Grazian et al. (2015). In the shallowest area, the incompleteness corrections as a function of magnitude have been estimated by comparing the observed galaxy number counts with those in the deepest HUDF area. For the other areas of intermediate depths we scaled the correction factors to fainter magnitudes taking into account the increasing $\mathrm{S} / \mathrm{N}$ ratio. Table 1 provides the magnitude corresponding to a $50 \%$ level of completeness in each of the areas covered by different sub-regions. The numbers of galaxies at $H<27$ are also shown in each region and at different redshifts.

Several catalogues of photometric redshifts have been provided for the CANDELS GOODS-S field. Dahlen et al. (2013) have made a comparison analysis among the various codes. Different galaxy templates have been used so that the codes are different enough to provide independent redshift estimates. They have combined independent estimates obtained by using nine different codes. Several spectroscopic redshifts available in the observed field were compared to the resulting photometric redshifts of the same objects to derive the best solution for the overall sample. The resulting average uncertainty is $|\Delta z| /(1+$ zspec $) \sim 0.03$ at relatively bright magnitudes $\left(H_{160} \leq 24\right)$, which increases to $\sim 0.06$ at $H_{160} \sim 26$ (Dahlen el al. 2013). The fraction of outliers (defined as differences $|\Delta z| /(1+z$ spec) $>0.15)$ is confined to $\sim 3 \%$ and in particular is low at $z>3.7$ owing to the Lyman continuum break signal. A Bayesian method has been developed to provide redshift probability distributions (PDFs) that can be useful for error estimates. The PDFs of the AGN candidates of the present sample are shown in the Appendix.

In total, there are 1113 galaxies in the GOODS-South field with a robust spectroscopic or photometric redshift at $z \geq 4$ which are analysed to search for any AGN activity. We note that the photometric redshifts have been estimated using theoretical spectral energy distributions derived from stellar populations only. No contribution from the non-stellar light typical of the AGN population has been included. Nevertheless, since the selection of faint AGNs is confined to $z \geq 4$, the redshift estimate is based on the dropout of the SED due to the neutral HI absorption by the IGM intervening along the line of sight, a feature independent of the main intrinsic spectral properties of the sources such as continuum spectral shape or escape fraction of ionizing photons from the AGN host galaxy. A large UV dropout is expected at $z>4$ by optically thick intervening absorption by the IGM even if the ionizing escape fraction from the AGN host galaxy is high. Recently Hsu et al. (2015) have provided a catalogue of photometric redshifts in the CANDELS GOODS-S area optimized for relatively bright X-ray detected AGNs. They add new optical intermediate-band filters to the CANDELS photometric catalogue and explore a mixing of galaxy and AGN templates. Their recipe appears to provide good results in terms of redshift accuracy and low fraction of outliers when compared with the spectroscopic redshift sub-sample. This is particularly true for bright sources with $H<23$. However, for our much fainter sample (typically $H=24-26$ ), the use of shallower intermediate band filters and mixed AGN/galaxy templates does not improve the accuracy of photometric redshifts and the fraction of outliers.

\section{The AGN selection}

The selection of AGN candidates relies on the selection of galaxies at $z>4$ having $H<27$ from the general CANDELS catalogue as described in the previous section. This corresponds to select sources on the basis of their detected rest-frame UV luminosity at $\lambda \sim 2000-3000 \AA$. Following the procedure proposed in Fiore et al. (2012) we searched for any sign of AGN activity by measuring the X-ray flux in the expected $H$ band position of the CANDELS sources having photometric or spectroscopic redshifts $z \geq 4$. In the following we briefly summarize the main steps. Since X-ray data are structured in event files, preserving time, spectral, and spatial information, a multidimensional source detection technique has been developed by Fiore et al. (2012) using spatial, spectral, and timing information. In particular, clustering of X-ray events in energy, time, and spatial coordinates was investigated to efficiently detect X-ray burst sources and X-ray sources characterized by specific features like strong lines or sharp edges, by reducing the elapsed time and/or the energy interval (i.e. the background) where the source detection is performed. Spectra were added at each galaxy position in different apertures from three to nine pixels. Sources are selected that have more than 12 background subtracted counts in the wide $0.3-4 \mathrm{keV}$ band. The background is evaluated from a map obtained from the original image after the exclusion of all bright sources. The background for each source was computed by normalizing an average background at the source offaxis to the source spectrum integrated in the $7-11 \mathrm{keV}$ energy band, where the source contribution is negligible owing to the decrease in the Chandra effective area. Source extraction aperture and contiguous energy band have been selected to minimize the Poisson probability for background fluctuation and to maximize the signal-to-noise ratio. Extensive simulated data have been performed using about $10^{5}$ background spectra at the positions of the detected sources using the same exposure time, PSF, etc., studying the number of spurious detections as a function of threshold, aperture, and energy band width. A combination of parameters has been adopted to keep the number of spurious detections smaller than one over 5000 spectra corresponding to a probability of spurious detection of $2 \times 10^{-4}$. This implies that for the 1113 galaxies at $z>4$ we expect only 0.2 spurious AGN candidates in the field. Finally, for each candidate position in the Chandra image we have checked that the accuracy of the relative astrometry is $\lesssim 1 \operatorname{arcsec}$ (see the appendix for an example).

We have detected 22 reliable AGN candidates with $z>4$ whose positions, redshifts, X-ray $(0.5-2 \mathrm{keV})$ fluxes, and $H$ magnitudes are shown in Table 2. Eleven of the detected sources are already in the Fiore et al. (2012) catalogue although with somewhat different photometric redshifts, four in the GOODS-S catalogues of X-ray selected sources by Luo et al. (2008), and seven are in Xue et al. (2011) (four in common with Luo et al. 2008). Five redshifts have been confirmed spectroscopically either by the presence of strong Lyman- $\alpha$ intervening absorption troughs (for the 9713 and 12130 objects observed in slitless low resolution spectroscopy with ACS camera at HST) or by the detection of Lyman- $\alpha$ emission. The detection of CIV at $z>4$ is usually not confirmed because out of the observed spectral region or expected in regions dominated by strong sky emission lines. The morphological appearance is indicated in Col. C where after visual inspection most candidates appear compact in $H$ band with $F W H M \sim 0.2-0.4$ arcsec. Only one (9945) has a complex morphology. The remaining are too faint for any classification. We note the larger number of AGN candidates emerging from the combined $H$ band selection plus X-ray detection 
Table 1. Area and magnitude limits of the CANDELS GOODS-South and HUDF fields.

\begin{tabular}{c|ccrr}
\hline \hline Field & $\begin{array}{c}\text { Area } \\
\operatorname{arcmin}^{2}\end{array}$ & $\begin{array}{c}H_{160} \text { Mag. compl. } \\
50 \%\end{array}$ & $\begin{array}{r}N_{\text {gal }} \\
H \leq 27\end{array}$ & $\begin{array}{r}N_{\text {gal }}^{\text {high-z }} \\
4 \leq z \leq 6.5\end{array}$ \\
\hline GOODS-South 1 & 11.05 & 25.75 & 801 & 24 \\
GOODS-South 2 & 25.03 & 26.00 & 2794 & 77 \\
GOODS-South 3 & 50.47 & 26.50 & 7984 & 240 \\
GOODS-South 4 & 77.18 & 27.00 & 15310 & 698 \\
GOODS-South 5 (HUDF) & 5.18 & 27.75 & 1672 & 74 \\
\hline Total & 168.91 & - & 26963 & 1113 \\
\hline
\end{tabular}

Table 2. Photometric and spectroscopic properties of the 22 AGN candidates.

\begin{tabular}{|c|c|c|c|c|c|c|c|c|c|c|c|}
\hline ID & RA & Dec & zphot & zspec & $\mathrm{C}$ & $H$ & $M_{1450}$ & $\begin{array}{c}\log F_{\mathrm{X}} \\
\mathrm{erg} / \mathrm{cm}^{2} / \mathrm{s}\end{array}$ & $\begin{array}{c}\log L_{X} \\
\text { erg/s }\end{array}$ & A & Previous catalogues \\
\hline 273 & 53.1220463 & -27.9387409 & 4.49 & $4.762^{1}$ & $\mathrm{c}$ & 23.96 & -21.37 & -15.97 & 43.80 & 2 & M208, X403 \\
\hline 4285 & 53.1664941 & -27.8716803 & 4.28 & & $\mathrm{cf}$ & 25.57 & -20.22 & -16.46 & 42.90 & 3 & - \\
\hline 4356 & 53.1465968 & -27.8709872 & 4.70 & & $\mathrm{cf}$ & 26.36 & -18.44 & -16.38 & 43.40 & 4 & M70437, L306, X485 \\
\hline 4952 & 53.1605007 & -27.8649890 & 4.32 & & $\mathrm{c}$ & 25.47 & -20.20 & -16.50 & 42.90 & 3 & - \\
\hline 5375 & 53.1026292 & -27.8606307 & 4.41 & & $\mathrm{c}$ & 25.16 & -20.16 & -16.66 & 42.75 & 4 & X331 \\
\hline 5501 & 53.1280240 & -27.8593930 & 5.39 & & $\mathrm{c}$ & 25.71 & -20.23 & -16.45 & 43.10 & 4 & - \\
\hline 8687 & 53.0868634 & -27.8295859 & 4.23 & & $\mathrm{c}$ & 26.90 & -19.19 & -16.43 & 42.90 & 4 & - \\
\hline 8884 & 53.1970699 & -27.8278566 & 4.52 & & $\mathrm{c}$ & 25.74 & -19.04 & -16.77 & 42.65 & 4 & - \\
\hline 9713 & 53.1715890 & -27.8208052 & 5.86 & $5.70^{2}$ & $\mathrm{c}$ & 26.54 & -19.87 & -16.46 & 43.15 & 4 & HUDF322 \\
\hline 9945 & 53.1619508 & -27.8190897 & 4.34 & $4.497^{3}$ & $\mathrm{~cd}$ & 24.99 & -20.93 & -16.65 & 42.75 & 4 & - \\
\hline 11287 & 53.0689924 & -27.8071692 & 4.94 & & $\mathrm{c}$ & 25.06 & -20.48 & -16.42 & 43.10 & 4 & M8728 \\
\hline 12130 & 53.1514304 & -27.7997601 & 4.43 & $4.62^{4}$ & $\mathrm{c}$ & 25.54 & -20.60 & -16.58 & 42.85 & 5 & HUDF3094 \\
\hline 14800 & 53.0211735 & -27.7823645 & 4.92 & $4.823^{5}$ & $\mathrm{c}$ & 23.43 & -22.51 & -16.38 & 43.10 & 3 & M10548 \\
\hline 16822 & 53.1115637 & -27.7677714 & 4.52 & & $\mathrm{c}$ & 25.67 & -18.97 & -15.91 & 43.85 & 4 & M70168, L245, X371 \\
\hline 19713 & 53.1198898 & -27.7430349 & 4.84 & & $\mathrm{c}$ & 25.31 & -18.14 & -16.48 & 43.00 & 4 & E1516, X392 \\
\hline 20765 & 53.1583449 & -27.7334854 & 5.23 & & $\mathrm{f}$ & 24.44 & -21.06 & -16.29 & 43.25 & 3 & E2551 \\
\hline 23757 & 53.2036444 & -27.7143907 & 4.13 & & $\mathrm{c}$ & 24.56 & -20.72 & -16.49 & 42.85 & 1 & - \\
\hline 28476 & 53.0646867 & -27.8625539 & 6.26 & & $\mathrm{f}$ & 26.77 & -19.03 & -16.60 & 43.10 & 4 & M70407 \\
\hline 29323 & 53.0409764 & -27.8376619 & 9.73 & & $\mathrm{cf}$ & 26.33 & -19.50 & -15.96 & 44.00 & 3 & M70340, L103, X156 \\
\hline 31334 & 53.2131871 & -27.7816486 & 4.73 & & $\mathrm{f}$ & 26.41 & -19.60 & -15.69 & 43.75 & 4 & - \\
\hline 33073 & 53.0547529 & -27.7368325 & 4.98 & & $\mathrm{c}$ & 26.89 & -19.19 & -16.44 & 43.10 & 2 & E2199 \\
\hline 33160 & 53.0062504 & -27.7340678 & 6.06 & & $\mathrm{cf}$ & 25.90 & -19.62 & -16.26 & 43.65 & 3 & E2498, L57, X85 \\
\hline
\end{tabular}

Notes. ID from Guo et al. (2013) catalogue. Photometric redshifts (zphot) from Dahlen et al. (2013) catalogue. Spectroscopic redshifts after: (1) Vanzella et al. (2008); (2) Hathi et al. (2008); (3) M. Dickinson \& D. Stern (priv. comm.); (4) Rhoads et al. (2009); (5) Balestra et al. (2010). Column $\mathrm{C}$ indicates the compactness of the sources: $\mathrm{c}=$ compact, $\mathrm{d}=$ diffuse, $\mathrm{f}=$ faint object. $\mathrm{X}$-ray fluxes are computed in the $0.5-2 \mathrm{keV}$ band and X-ray luminosities in the $2-10 \mathrm{keV}$ band. M,E in previous catalogues are from Fiore et al. (2012), L from Luo et al. (2008), X from Xue et al. (2011). Column A indicates the GOODS-S field area according to Table 1.

respect to the direct X-ray selection. X-ray luminosities have been computed for the brightest sources by adopting a standard single power law with fixed slope 1.8 of the power-law photon index and fitting a gas absorption. We derived hydrogen column densities of $\log N_{\mathrm{H}} \sim 23.5$ for $273,4356,16822,29323,33160$ and $\log N_{\mathrm{H}} \sim 22.5$ for 33073,20765 . For the remaining X-ray fainter objects we adopted $\log N_{H} \sim 22$. The reliability of the estimated X-ray absorption derived for the estimate of X-ray fluxes and luminosities in $z>4$ faint sources is affected by the higher rest-frame energy sampled, by the adopted slope in the power-law fitting, and by other physical assumptions like covering factor and metallicity of the absorbing gas, as discussed in Sect. 6. The derived X-ray luminosities in Table 2 have an average value of $\log L_{\mathrm{X}} \sim 43.2 \mathrm{erg} \mathrm{s}^{-1}$ and are more typical of Seyfert-like sources than of starburst galaxies, although we cannot exclude a significant contribution from stars to the X-ray luminosity of some peculiar source in our sample. Analysis by Fiore et al. (2012) of the brightest sources at $z \sim 3$ suggests that the possible presence of significant X-ray absorption is uncorrelated with the presence of Lyman- $\alpha$ and CIV emission lines and low dust extinctions as in the case where absorption is in general close to the emitting ionized region and inside the sublimation radius.

Figure 1 shows as an example the images of two sources with decreasing wavelength from the IR Spitzer images to the HST images, as well as the X-ray image. The first source on the left, 16822 , has a good signal-to-noise ratio and appears compact in the $H$ band. The second AGN candidate on the right, 28476 , is among the highest redshift sources in our sample. It is much fainter in the $H$ band and lacks morphological information.

Figure 2 shows the spectral energy distributions of the two candidates shown in Fig. 1 together with their galaxy spectral fits and the derived photometric redshifts. We note how faint the selected AGNs are in the optical bands. The SEDs of all the selected AGN candidates are shown in the Appendix. This emphasizes the importance of the joint $H$ band, X-ray detections to find faint AGNs in multiwavelength surveys (Fiore et al. 2012). Figure 3 shows the $\mathrm{X}$-ray over $H$ band flux ratio as a function of the $H$ band magnitude for our CANDELS GOODS-S sample of AGN candidates. The straight line in the figure represents 

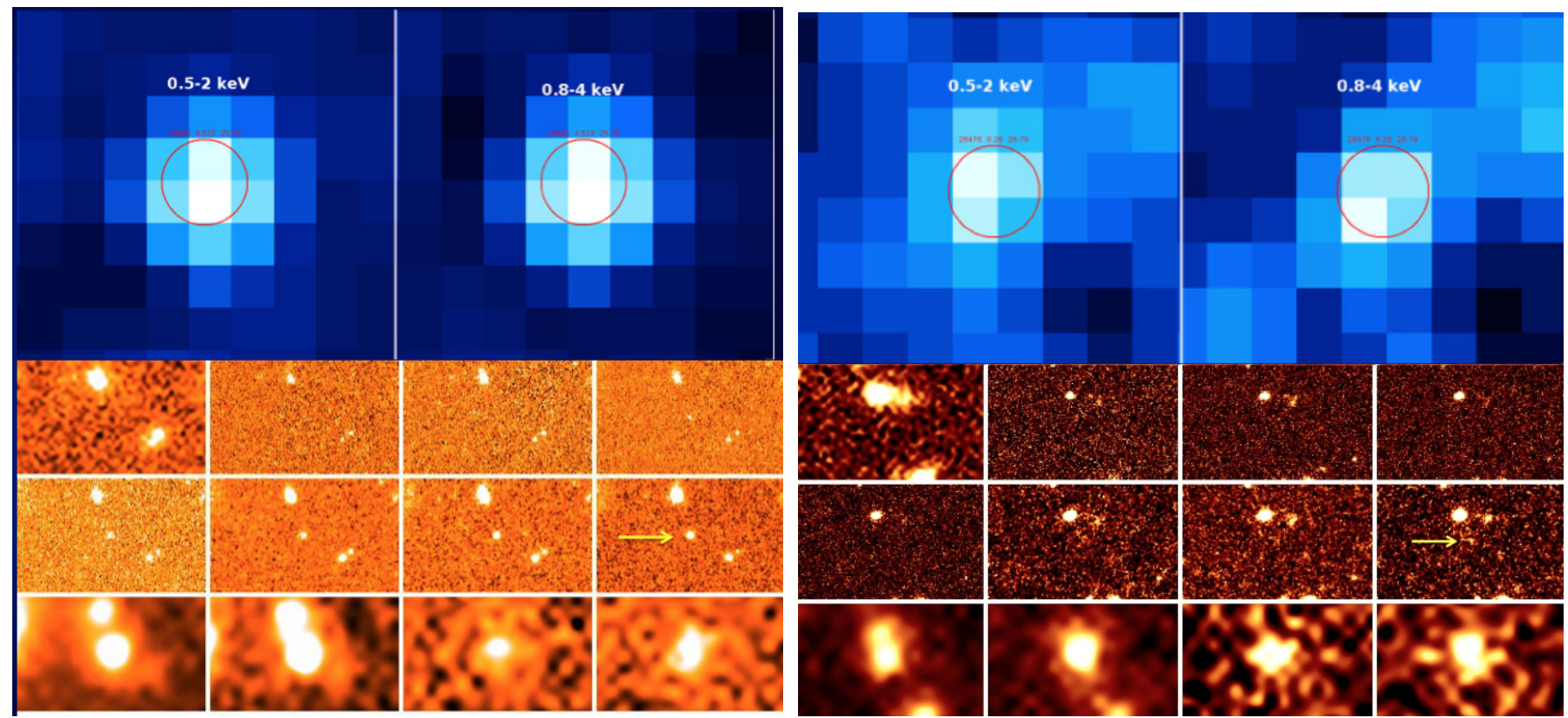

Fig. 1. Images of two AGN candidates. From left to right the filters are $\mathrm{X}_{0.5-2}, \mathrm{X}_{0.8-4}, \mathrm{U}_{,} \mathrm{B}_{435}, \mathrm{~V}_{606}, \mathrm{I}_{775}, \mathrm{Z}_{850}, \mathrm{Y}_{1.05}, \mathrm{~J}_{1.25}, \mathrm{H}_{1.6}$, and IRAC $\mathrm{IR}_{3.6}, 4.5,5.8,8$. Left box: object 16822 at $z=4.5$. Right box: object 28476 at $z=6.26$. The size of the optical/IR image is $9 \times 6$ arcsec ${ }^{2}$. Red circles in the X-ray images show the expected position from the $H$ band detection with a circular size of 1 arcsec.
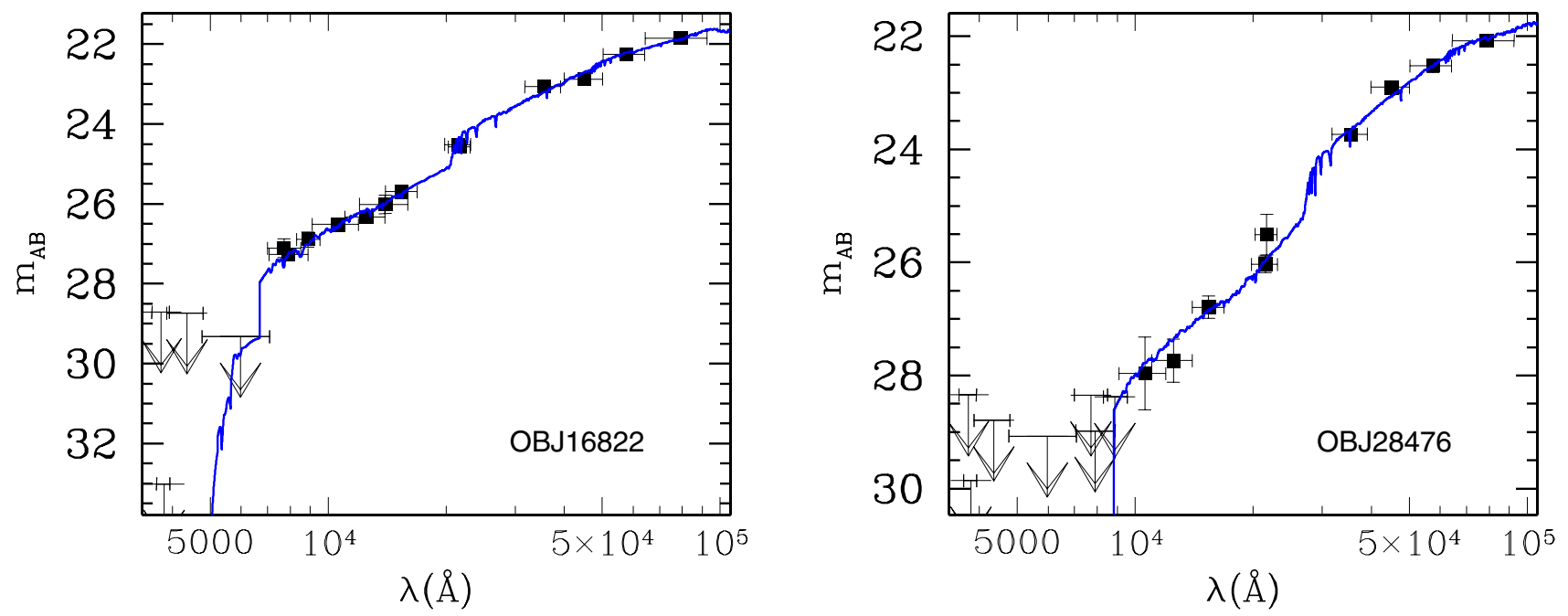

Fig. 2. Spectral energy distributions of the objects as in Fig. 1.

the locus of constant X-ray flux corresponding to the average detection threshold. Looking at the $X / H$ ratio distribution derived at different $H$ magnitudes, it appears that only faint NIR $z \geq 4$ candidates that are relatively bright in the X-ray band can be detected in the Chandra 4 Ms field. In this respect our sample is biased against relatively X-ray faint AGNs, a feature that has to be taken into account in estimating the faint end of the AGN UV luminosity function as computed in the next section. We note again that our sample comes from a well-defined magnitude selected galaxy sample in the UV rest-frame band. The $\mathrm{X}$-ray detection is only used in this context to reveal the presence of AGN activity.

\section{AGN UV luminosity functions}

The estimate of the faint end of the AGN luminosity function in the UV rest frame has been performed with an extended version of the standard $1 / V_{\max }$ algorithm (Schmidt 1968) where different regions in the GOODS-S field with different magnitude limits were combined together in the volume estimate of each object (e.g. Avni \& Bahcall 1980). Indeed, for each object and for each $j$ th region shown in Table 1, an effective volume $V_{\max }(j)$ is computed. For a given redshift interval $\left(z_{\text {low }}, z_{\text {up }}\right)$, these volumes are confined between $z_{\text {low }}$ and $z_{\lim }(j)$, the latter being defined as the minimum between $z_{\text {up }}$ and the maximum redshift at which the object could have been observed within the magnitude limit of the $j$ th region. The galaxy number density $\phi(M, z)$ in a given $(\Delta z, \Delta M)$ bin can be computed as

$\phi(M, z)=\frac{1}{\Delta M} \sum_{i=1}^{n}\left[\sum_{j} \omega(j) \int_{z_{\mathrm{low}}}^{z \mathrm{lim}(i, j)} \frac{\mathrm{d} V}{\mathrm{~d} z} \mathrm{~d} z\right]^{-1}$,

where $\omega(j)$ is the area in units of steradians corresponding to the region $j$ shown in Table $1, n$ is the number of objects in the bin, and $\mathrm{d} V / \mathrm{d} z$ is the comoving volume element per steradian (see e.g. Salimbeni et al. 2008). 


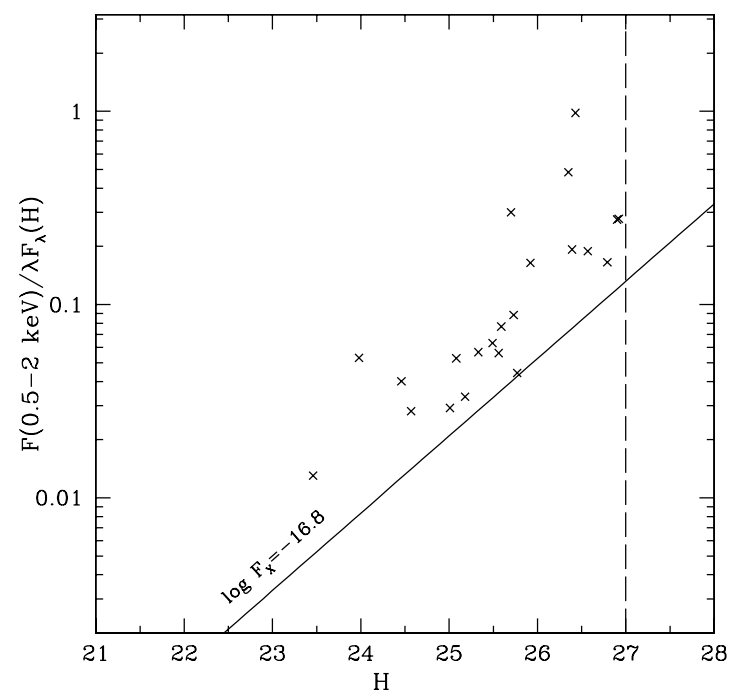

Fig. 3. $X / H$ flux ratio as a function of $H$ magnitude for the CANDELS GOODS-S sample. The X-ray flux limit adopted in the Chandra image is also shown, together with the $H$ magnitude limit $H=27$.

Rest frame UV $1450 \AA$ absolute magnitudes $M_{1450}$ were computed from apparent magnitudes in the filters closer to this rest frame wavelength for each redshift. For example, for AGNs at $z \sim 4.5$ the UV rest-frame absolute magnitudes have been directly derived from the observed HST $i_{775}$ magnitudes. The absolute magnitudes $M_{1450}$ used for the evaluation of the luminosity function are shown for each object in Table 2.

The resulting volume densities as a function of $M_{1450}, z$ have then been corrected for various sources of incompleteness. The first correction is due to the gradual $H$ band bending of the galaxy counts which corresponds to a 50\% drop at $H=27$ in area No. 4 of the present sample. In this respect, a correction factor has been included as a function of area and $H$ magnitude in the volume estimate of each object. The second correction is due to the fact that, for each $H$ magnitude, only relatively bright X-ray AGN candidates (with $f_{\mathrm{X}}>1.5 \times 10^{-17} \mathrm{erg} \mathrm{s}^{-1} \mathrm{~cm}^{-2}$ ) can be detected in our $z \geq 4$ parent sample (see Fig. 3). This corresponds to the loss of AGN candidates with e.g. $F_{\mathrm{X}} /\left(\lambda f_{H}\right) \lesssim 0.1$ at $H \sim 27$. The incompleteness fraction is derived from the same $X / H$ distribution observed above the X-ray flux threshold. The objects have been weighted considering that their $H$ distribution in Fig. 3 is affected by the faint slope of the UV rest-frame luminosity function and by the incompleteness in the $H$ band counts. We adopted a faint end slope of -1.5 and a count incompleteness as derived in Sect. 2. The two effects tend to compensate and the final correction is not sensitive to slope changes of $\sim 0.2$. Finally, correction factors to volume densities typically of $10-20 \%$ have been applied to volume densities after taking into account spatial fluctuations in the X-ray flux limits for each position of the $\mathrm{X}$-ray detection.

Given the very poor statistics we only evaluated the Poisson contribution to the errors in each LF bin adopting the recipe by Gehrels (1986), which is also valid for small numbers. In this respect the errors shown in the figure represent lower limits than the true values. For example, cosmic variance can play a significant role in the present deep pencil beam survey by increasing the uncertainties in the derived volume densities.

The resulting luminosity functions are shown in Fig. 4 and Table 3 for $M_{1450} \leq-18.5$ where the width and position of the bins have been selected to allow where possible homogeneous statistics among the bins. It is clear that our faint AGN candidates are sampling the faint end of the AGN UV luminosity function in the absolute magnitude range $-18.5 \gtrsim M_{1450} \gtrsim$ -22.5 and with densities at the faint end between $10^{-5} \lesssim \phi \lesssim$ $10^{-4} \mathrm{Mpc}^{-3} \mathrm{mag}^{-1}$. Thus, the presence of even a few faint high- $z$ AGN candidates in the GOODS-S field appreciably changes the abundance of the AGN population at $z=4-6.5$ and its cosmological significance especially in the context of the cosmic reionization.

To provide a first estimate of the total AGN emissivity at $z \geq 4$ a shape of the luminosity function has been derived in each redshift bin connecting the volume densities estimated from our sample with that of the brightest high- $z$ SLOAN QSO sample where selection effects with respect to the morphological appearance and X-ray properties are thought to be small. In other words, no X-ray QSOs with strong absorption in the rest-frame optical/UV are expected at the brightest magnitudes where the Sloan sample should be representative of the overall AGN population. We adopted a double power-law shape to connect the two samples in each redshift bin of the type

$\phi=\frac{\phi^{*}}{10^{0.4\left(M_{\text {break }}-M\right)(\beta-1)}+10^{0.4\left(M_{\text {break }}-M\right)(\gamma-1)}}$.

The best fit parameters are shown in Table 3. We note that in the highest redshift bin the faint end slope and the break magnitude have been fixed to the average values in the two lower redshift intervals because of the lower statistics. In general, however, it is clear from Fig. 4 that the break magnitude and the related volume density are not well constrained from the present composite sample. For this reason, the fitted volume density at the break is likely a lower limit and larger AGN surveys of intermediate depth are needed to fix the point. In the lowest redshift bin $4 \leq z<4.5$ we have also shown for comparison the points of the luminosity function derived from a faint sample of type 1 QSOs with stellar appearance by Glikman et al. (2011). We note how the X-ray detection criterion used in the present paper is able to reach higher volume densities including AGNs with different morphological and spectral properties. In the highest redshift bin $5 \leq z<6.5$ we have also shown in Fig. 4 the recent point by Kashikawa et al. (2015) obtained from an optical spectroscopic survey where AGN candidates are selected from standard colour selection, assuming a stellar appearance. We have added a vertical arrow to note that this value could represent a lower limit than the combined NIR/X-ray surveys that are presented here. A decrease in volume density by a factor of $\lesssim 2$ from $z \sim 4.25$ to $z \sim 5.75$ is present in our sample, smaller than found at higher luminosities in UV and X-ray selected surveys (e.g. Vito et al. 2014). In summary, our new data give a first estimate of the overall shape of the UV AGN luminosity function in the absolute magnitude interval $-18.5 \gtrsim M_{1450} \gtrsim-28$. A flattening of the LF is needed to match the volume density of the brightest QSOs derived from the Sloan survey to that of our faint AGN population. The global behaviour of the luminosity function is used in the next section to derive the high redshift evolution of the UV emissivity.

\section{AGN hydrogen ionizing emissivity and photoionization rate}

We now compute the contribution of our predicted luminosity functions to the AGN hydrogen ionizing emissivity. This is 
E. Giallongo et al.: Faint AGNs at $z>4$ in the CANDELS GOODS-S field

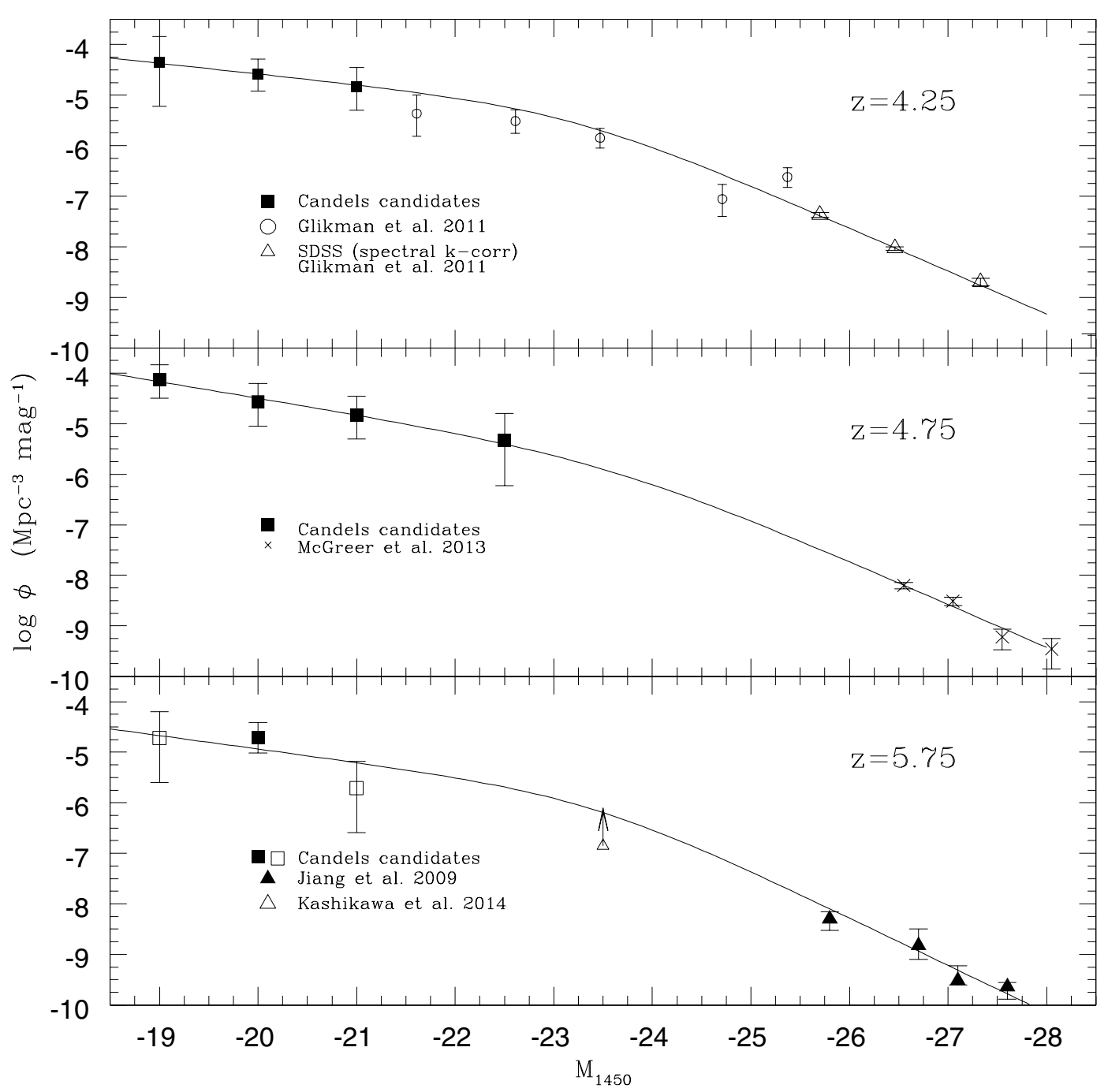

Fig. 4. UV $1450 \AA$ AGN luminosity functions in various redshift bins. Different symbols represent different surveys as explained in the inset. Open squares in the highest redshift interval (bottom panel) represent LF bins derived from AGN candidates with more uncertain photometric redshifts (see Sect. 6.1).

given by

$$
\begin{aligned}
\epsilon_{\text {ion }}(z) & =\langle f\rangle \epsilon_{912} \\
& =\langle f\rangle \int \phi\left(L_{1450}, z\right) L_{1450}\left(\frac{1200}{1450}\right)^{0.44}\left(\frac{912}{1200}\right)^{1.57} \mathrm{~d} L_{1450},
\end{aligned}
$$

where $\langle f\rangle$ is the average escape fraction of ionizing radiation from the AGN host galaxies and $\epsilon_{912}$ is the emissivity produced by AGN activity. The shape of the AGN SED from $\lambda=1450 \AA$ to $\lambda=912 \AA$ has been represented by a double power law with slopes adopted from Schirber \& Bullock (2003), Telfer et al. (2002), and Vanden Berk et al. (2001). In the integral we have considered all the AGNs that are a factor of 100 brighter or fainter than the break luminosity (corresponding to a faint limit of $M_{1450} \sim-18$ ). Considering sources even 100 times fainter would only increase the emissivity by a factor of $\sim 10 \%$ given the rather flat slope of the luminosity function. The uncertainties in the integrated emissivities are computed combining the uncertainties in the normalization and bright end slope for the highest redshift bin since the faint end slope and break have been fixed. For the lower redshift bins the uncertainties are due to uncertainties on the normalization and faint end slope only (i.e. fixing the break luminosity and bright end slope). In this respect the widths of the error bars in emissivity and photoionization rates should be considered as lower limits. We note that in the sampled redshift intervals the main contribution to the emissivity comes from AGNs near the predicted break magnitude $M_{1450} \sim-23.5$. It is just at this magnitude where volume densities should be estimated with greater accuracy, but this requires surveys of comparable depth extended over larger areas.

In Fig. 5 and Table 3 the Lyman continuum ionizing emissivities $\epsilon_{24}$ in units of $10^{24} \mathrm{erg} \mathrm{s}^{-1} \mathrm{~Hz}^{-1} \mathrm{Mpc}^{-3}$ derived from the estimated AGN luminosity functions are shown as a function of redshift. We assume $\langle f\rangle=1$ as a reference value for the ionizing escape fraction as observed in most bright AGNs.

The prediction of the model proposed by Giallongo et al. (2012) is also shown in Fig. 5 for comparison. The model is based on the standard $\Lambda$ CDM scenario for galaxy formation and evolution already described in Menci et al. (2005) and includes a physical description of the super massive black hole growth at the centre of galaxies as mainly due to the inflow of cold gas destabilized by minor merger events (Cavaliere \& Vittorini 2000; Menci et al. 2008). The model also includes a self consistent description of the ionizing emissivity of AGNs based on 
Table 3. AGN luminosity functions, emissivities, and photoionization rates.

\begin{tabular}{cccccccccccc}
\hline \hline$\Delta z$ & $M_{1450}$ & $\log \phi_{\text {obs }}$ & $\log \phi_{\text {corr }}$ & $N_{\text {obj }}$ & $\beta$ & $\gamma$ & $M_{\text {break }}$ & $\log \phi^{*}$ & $\epsilon_{1450}$ & $\epsilon_{912}$ & $\Gamma$ \\
\hline $4-4.5$ & & & & & 1.52 & 3.13 & -23.2 & -5.2 & 11.45 & 6.8 & 0.62 \\
& -19 & -4.9 & -4.3 & 1 & & & & & & & \\
& -20 & -4.7 & -4.6 & 3 & & & & & & & \\
& -21 & -4.9 & -4.9 & 2 & & & & & & & \\
\hline $4.5-5$ & & & & & 1.81 & 3.14 & -23.6 & -5.7 & 9.90 & 5.92 & 0.43 \\
& -19 & -4.5 & -4.1 & 3 & & & & & & & \\
& -20 & -4.9 & -4.6 & 2 & & & & & & & \\
& -21 & -4.9 & -4.8 & 2 & & & & & & & \\
& -22.5 & -5.3 & -5.3 & 1 & & & & & & \\
\hline $5-6.5$ & & & & & $1.66^{a}$ & 3.35 & $-23.4^{a}$ & -5.8 & 4.2 & 2.5 & 0.12 \\
& -19 & -5.2 & -4.7 & 1 & & & & & & & \\
& -20 & -5.1 & -4.7 & 3 & & & & & & & \\
& -21 & -5.7 & -5.7 & 1 & & & & & & & \\
\hline
\end{tabular}

Notes. $\phi$ in units of $\mathrm{Mpc}^{-3}, \epsilon$ in units of $10^{24} \mathrm{ergs} \mathrm{s}^{-1} \mathrm{~Hz}^{-1} \mathrm{Mpc}^{-3}, \Gamma$ in units of $10^{-12} \mathrm{~s}^{-1} . \phi_{\text {corr }}$ is the volume corrected for incompleteness in the $X / H$ distribution. ${ }^{(a)}$ Value has been fixed to average in the two lower $z$ intervals.

the blast-wave feedback mechanism (Cavaliere et al. 2002; Lapi et al. 2005). The blast-wave produced in the interstellar medium sweeps out the gas allowing ionizing UV photons to escape from the AGN host galaxy into the IGM. The model prediction tends to overestimate the observed emissivities by factors of two to five as the redshift increases from $z \sim 4$ to $z \sim 6.5$. In the same Fig. 5 the AGN model prediction by Haardt \& Madau (2012) is also shown for comparison. The latter model underestimates by a larger amount the observed values up to a factor of 20 at $z \sim 6$. Overall, the new data are in between the two models, not far from the predictions of the Giallongo et al. (2012) model, and show a gradual decline of the ionized emissivity in the probed redshift interval $z=4-6.5$. To see whether this decline is consistent with the evolution of the IGM ionization level we computed the hydrogen photoionization rate predicted from our emissivity and compared it with that inferred from the analysis of the Lyman- $\alpha$ forest in high redshift QSO spectra.

The photoionization rate per hydrogen atom $\Gamma_{-12}$ in units of $10^{-12} \mathrm{~s}^{-1}$ was computed as in Giallongo et al. (2012) including the recent update on the mean free path $(\mathrm{mfp})$ of ionizing photons in the IGM derived by Worseck et al. (2014a),

$\Gamma_{-12}(z) \simeq 0.6 \frac{\epsilon_{24}(z)}{3+\left|\alpha_{\mathrm{UV}}\right|}\left(\frac{\Delta l}{65 \mathrm{Mpc}}\right)\left(\frac{1+z}{4.5}\right)^{3-\eta}$,

where $\alpha_{\mathrm{UV}}=-1.57$ (Schirber \& Bullock 2003). The value $\alpha_{v}=-1.57$ adopted for $475<\lambda<912 \AA$ is consistent with the recent evaluation with the COS spectrograph at HST by Shull et al. (2012) and Stevans et al. (2014) $\alpha_{v}=-1.41$. The powerlaw index $-\eta$ describes the decrease in redshift of the proper mfp $\Delta l$ of ionizing photons in the IGM due to the increase in redshift of the Lyman limit absorption systems. We adopt $\eta=5.4$ and the normalization of the mfp to $65 \mathrm{Mpc}$ at $z=3.5$ as found by Worseck et al. (2014a) in the redshift range $z=4.4-5.5$. We note that the equation assumes that only ionizing sources within one absorption length contribute to the cosmic reionization. The values derived from our CANDELS GOODS-S sample of AGN candidates show (see Fig. 6) a gradual decline from $z=4$ to $z=6.5$ although slightly steeper than that derived for the emissivity due to the increase of the average IGM absorption with increasing redshift.

Different data points derived in a model-dependent way from the IGM statistics observed in the Lyman- $\alpha$ forest of high- $z$ QSO spectra are also shown for comparison as in Giallongo et al. (2012). Some values depend on the UV background adopted in numerical simulations to reproduce the mean flux decrement observed in the Lyman- $\alpha$ forest of QSO spectra (Faucher-Giguere et al. 2008; Bolton et al. 2005; Becker \& Bolton 2013; Fan et al. 2006; Wyithe \& Bolton 2011). Other values are derived from the analysis of the proximity effect at the highest redshifts $z \sim 5-6$ (Calverley et al. 2011). Different methods produce the significant scatter shown in Fig. 6 although the data are scaled to the same IGM temperature-density relation as in Giallongo et al. (2012). This is due to uncertainties on modelling the gas density and temperature distributions in the IGM at very low densities (Miralda-Escudé et al. 2000) and within the proximity region surrounding each QSO (Calverley et al. 2011).

It is important to note in Fig. 6 that the photoionization rates at $z>4$ derived by our AGN luminosity functions are consistent with the photoionization levels of the IGM derived from the Lyman- $\alpha$ statistics in the same redshift range. This would imply a reionization occurring at redshifts not much larger than $z \sim 7$ since moderately bright ionizing sources with a maximum comoving density of $\sim 2 \times 10^{-5} \mathrm{Mpc}^{-3}$ at $z \sim 6$ have a typical separation of $\sim 3$ proper Mpc, only a factor of 2 smaller than the derived proper mean free path of ionizing photons in the IGM at $z \sim 6$ by Worseck et al. (2014a). The latter scenario would also be consistent with the large decline of strong Lyman- $\alpha$ emitting galaxies observed between $z \sim 6$ and $z \sim 7$ which would suggest a large neutral hydrogen fraction in the IGM and a patchy reionization process already at $z \sim 7$ (Pentericci et al. 2011; Treu et al. 2013; Pentericci et al. 2014; Tilvi et al. 2014).

\section{Discussion}

In the context of this preliminary analysis we should discuss some caveats which could significantly reduce our expectations about the contribution to the reionization by high redshift AGNs of intermediate-low luminosities. First of all some redshift estimates for our candidates could be incorrect, despite the average high level of accuracy, reducing the number of high- $z$ AGNs. The second issue concerns the assumption about the predominance of the AGN UV spectral energy distribution even in cases where the optical spectra appear to be affected by significant stellar contribution or look very steep. A further issue concerns 


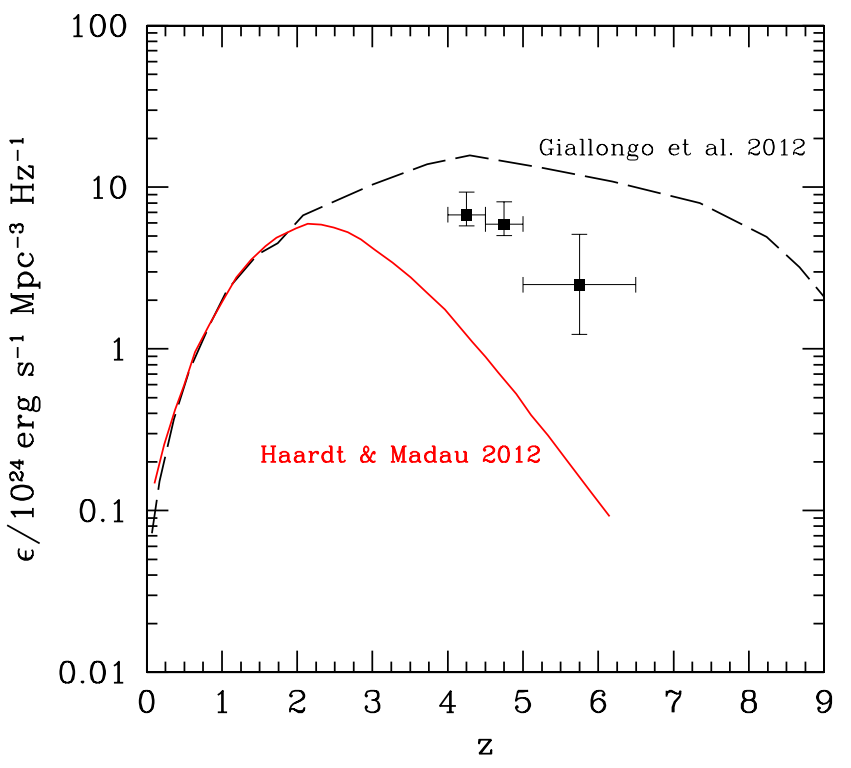

Fig. 5. Cosmic ionizing emissivity by AGNs as a function of redshift assuming $\langle f\rangle=1$. Black squares are from our sample. The red continuous curve is from the Haardt \& Madau (2012) model. The long dashed curve is from the Giallongo et al. (2012) model.
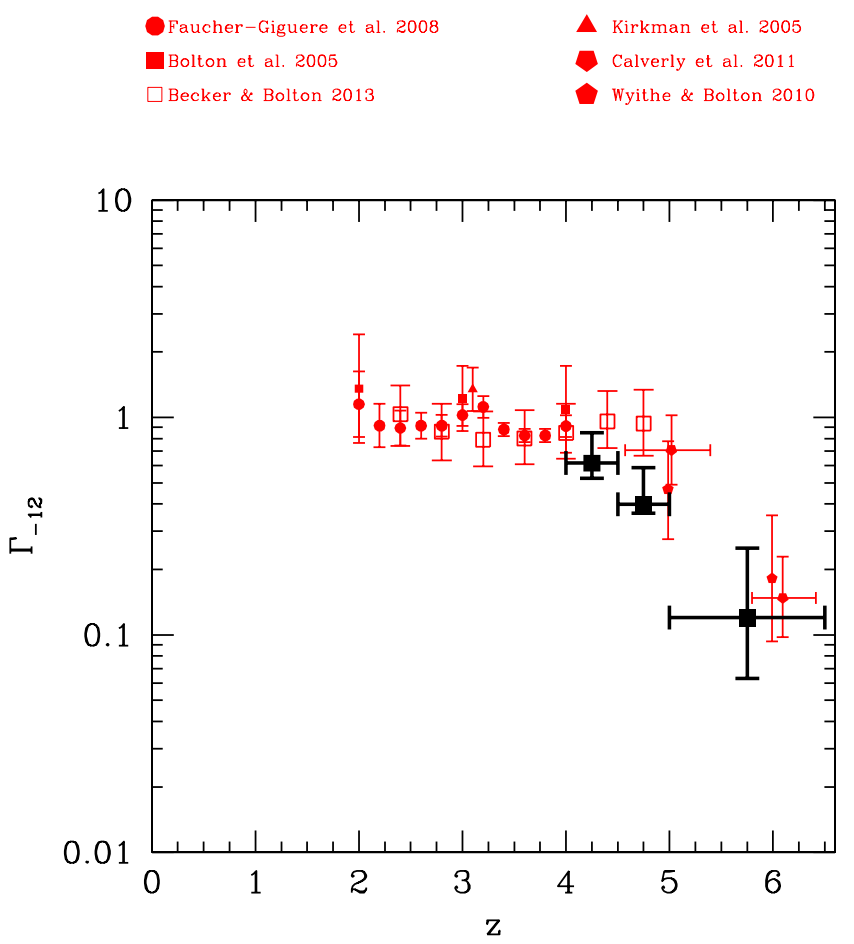

Fig. 6. Cosmic ionizing photoionization rate $\Gamma_{-12}$ in units of $10^{-12} \mathrm{~s}^{-1}$ produced by AGNs as a function of redshift assuming $\langle f\rangle=1$. Black filled squares represent the predicted contribution by faint AGNs from the GOODS-S sample. Other small red symbols are the values inferred from the ionization status of the IGM as derived from the Lyman- $\alpha$ forest analysis in high- $z$ QSO spectra.

the assumption of a high escape fraction of ionizing photons for the global AGN population, including the X-ray absorbed AGN fraction. Finally we discuss the implications that an ionizing AGN population at very high redshift would have for an early HeII reionization of the IGM.

\subsection{Redshift reliability}

Concerning the reliability of the derived redshift distribution in our sample we should note that the evaluation of the photometric redshifts becomes progressively more uncertain for fainter sources with featureless SEDs. For this reason we have shown in the Appendix the probability redshift distributions $\operatorname{PDF}(\mathrm{z})$ for our candidates. In this context, as already stated, the estimate of photometric redshifts for sources at $z>4$ mainly relies on the statistical significance of the Lyman- $\alpha$ forest $(<1216 \AA)$ and Lyman break at $912 \AA$ rest frame wavelength. In fact, the expected escaping Lyman continuum emission from the sources, even assuming $\langle f\rangle \simeq 1$, would be strongly depressed by IGM absorption causing a flux dropout. As a consequence, the redshift uncertainty at $z=5-6$ is related to the flux dropouts near the Lyman- $\alpha$ and Lyman edge almost independently of the assumed galaxy or AGN spectral library. Our assumption is corroborated by the good agreement we found between the five available spectroscopic redshifts and the photometric estimates of the CANDELS catalogue. It is clear, however, that in cases where the spectrum is particularly steep the evidence for the presence of a Lyman break weakens, increasing consequently the uncertainty in the redshift estimate. This is particularly true for some of the $z>5$ objects. We have already excluded object 29323 from the LF analysis because of its peculiar SED and $\operatorname{PDF}(z)$. Thus, five sources have been used for the LF estimate in the highest redshift bin, among them 20765, 28476 , and 33160 have the most uncertain redshift estimates as shown in Fig. A.1. To get a rough estimate of the uncertainties involved in the derived volume densities of faint AGNs at $z>5$ we have repeated the estimate of the luminosity function at the highest redshift bin excluding these sources. Two of them are the only sources in the faintest and brightest LF bins $\left(M_{1450}=-19\right.$ and $\left.M_{1450}=-21\right)$ that would be removed. We have indicated with different symbols these uncertain LF bins. Two sources remain in the LF bin at $M_{1450}=-20$ slightly decreasing the average volume densities by -0.1 to $\log \phi=-4.8$. This value would still be consistent with the double power law extrapolation adopted to estimate the UV emissivity. Finally, we note that some of these sources are relatively bright at 8 microns, but this by no means represents a prior against a high redshift solution for the estimated redshift. One of the best studied X-ray absorbed faint AGNs in our catalogue, 273 (e.g. Vanzella et al. 2008), which has also been observed by ALMA (Gilli et al. 2014), has a robust spectroscopic redshift at $z=4.76$ and an 8 micron apparent $\mathrm{AB}$ magnitude of 21.5. Source 14800 at spectroscopic redshift $z=4.82$ also shows a relatively bright IRAC continuum at levels of 22.5 .

Very recently Weigel et al. (2015) searched for $z>5$ AGNs in the same GOODS-S field using almost the same CANDELS dataset and found no convincing AGN candidates. The different result depends on their adopted procedure. They looked for $z>5$ sources starting from the Xue et al. (2011) X-ray selected catalogue, looking for plausible optical drop-outs and/or photometric redshifts in the CANDELS images. Our sample is based on NIR selection in the $H$ band and reaches fainter X-ray fluxes than the Xue et al. (2011) catalogue. Moreover photometric redshifts in our sample have been obtained from the CANDELS multiwavelength catalogue derived from the $B$ to the 8-micron Spitzer band that included careful Spitzer deblended photometry. As an example one source in our sample (9713, not included in the Xue et al. 2011 sample) has a spectroscopic confirmation at $z=5.7$. In our sample of 22 AGN candidates only two are in common with the Xue et al. (2011) catalogue with an estimated redshift $z>5$. One of the two, 29323 (X156), has already been 
excluded from our analysis as stated above. The second object, 33160 (X85), appears at $z \sim 6$ although with a larger uncertainty and we have already mentioned in the previous paragraph the effects of its removal from the sample.

\subsection{UV spectral shape}

With few broadband filters that often show only upper limits it is impossible to disentangle the relative AGN and stellar contributions to the SED of our faint $H=24-27$ mag sources. This is why we do not include AGN candidates at $z<4$ in our sample. Only at very high redshifts $(z=4-6)$ the photometric redshifts are mainly constrained by strong Lyman absorptions by the intergalactic medium intervening along the line of sight, almost independently of the intrinsic spectral shape assumed (AGN or galaxy template). If the sources are at very high redshifts, the observed optical bands ( $<1$ micron) give access to the rest frame UV SED shortward of $3000 \AA$. The presence of X-ray detection in our sample at levels $\log L_{\mathrm{X}} \gtrsim 43\left(\mathrm{erg} \mathrm{s}^{-1}\right)$ implies probable AGN emission. As a consequence, somewhere in the UV part of the spectrum the AGN emission should emerge above the stellar spectrum. We assume that this happens at wavelengths just shortward of $1500 \AA$. In particular we assume that our $1450 \AA$ luminosity is mainly due to AGN emission. Of course to verify this hypothesis detailed spectroscopic information would be required that is beyond the capability of the present instrumentation because of the faintness of our candidates. Nevertheless, this assumption is supported by the distribution of the rest frame $\mathrm{X} / \mathrm{UV}$ flux ratio in our sample (which at $z>4$ corresponds to the $X / H$ ratio distribution shown in Fig. 3). Our AGN candidates are confined in the $X / H$ interval between about 0.01 and $0.3-0.5$ (in the same units of Fig. 3) usually occupied by the AGN population in brighter AGN surveys as shown for example in Fiore et al. (2012) for the COSMOS high redshift AGNs (Civano et al. 2011). The few SLOAN QSOs at $z=6$ also show $X / H$ fluxes that occupy the same region up to $0.2-0.3$, although most of them have ratios below 0.1 because they are very bright optically selected sources (and so with a significant X-ray weak fraction). If the rest frame UV fluxes were dominated by stellar emission this would imply intrinsic AGN $X / H$ flux ratios much greater ( $>10$ times) than generally observed in brighter high redshift AGNs. Of course both rest-frame X-ray and UV fluxes could be produced by stellar populations, but this seems unlikely for X-ray luminosities $\log L_{X} \gtrsim 43$ ( $\mathrm{erg} \mathrm{s}^{-1}$ ). The suggestion provided by the SED models about the possible presence of Balmer breaks is not compelling and is not necessarily caused by stars. Rest-frame optical nebular emission lines (at $z=5-6$ ) could mimick Balmer breaks whose contribution to broadband filters increases with $(1+z)$ as noted by Shaerer $\&$ de Barros (2009).

Finally, to check if the average spectral shape assumed to estimate the ionizing emissivity from the UV AGN luminosity function is appropriate for our sample, we have derived a first rough guess of the average power-law slope in the UV rest-frame interval 1300-1700 $\AA$, just around $1500 \AA$. The average slopes have been derived from the $V, I$, and $z$ bands at $z \sim 4$ and the $Y$, and $J$ bands at $z \sim 6$. The average (median) slope $\alpha_{v}$ excluding object 9323 was found to be $-1.4(-1.2)$, although the dispersion is significant. In our paper we have adopted a flatter slope from 1450 down to $1200 \AA(-0.44)$ and a steeper one shortward of $1200 \AA(-1.57)$. Assuming the derived average slope -1.4 (instead of -0.44) just shortward of $1450 \AA$ reduces the photoionization rates by only about 10 per cent. To halve the derived photoionization rates in Fig. 6 we should adopt a much steeper -2.5 slope for all the AGNs in the luminosity function. The average UV slope derived in our sample (-1.4) appears to be consistent with the value $(-1.57)$ adopted in the paper for the overall AGN population from $M_{1450}=-18$ to $M_{1450}=-28$.

In summary, although we cannot reliably quantify the stellar contribution to the optical/UV emission we assume that in our X-ray sources the SED shortward of the rest-frame $1450 \AA$ is dominated by AGN emission. This assumption is consistent with the typical AGN $X / H$ (X/UV rest-frame) flux ratio found in our sample, which would imply an average UV-X spectral slope similar to that found in AGNs.

\subsection{Ionizing escape fraction}

One of the main uncertainties concerns the assumption about the escape fraction of UV photons from AGNs. In fact, we assume that all ionizing photons are able to escape and ionize the surrounding IGM. This is usually almost true for bright type 1 QSOs where the optical depth is $\tau_{\mathrm{LL}}<1$ at the Lyman limit position corresponding to the systemic redshift of the QSOs. Any appreciable absorption is present at shorter wavelengths because of the intervening IGM along the line of sight (e.g. Sargent et al. 1989). Our faint sample of AGN candidates lacks spectroscopic information except for the five cases shown in Table 2 and could include a significant fraction of absorbed AGNs. Hints of excess absorption have been derived in the X-ray spectral analysis of high redshift X-ray selected AGNs, but the reliability of any measure is hampered by the fact that the photoelectric cut-off of the absorption goes out of the spectral region sampled by the Chandra observations already at $z>3$ (see e.g. Vito et al. 2013). Moreover, to allow a first estimate of the hydrogen column density with few photon counts, a fixed slope (typically $\Gamma=1.8$ ) of the intrinsic X-ray power-law is often adopted and a solar metallicity is always assumed despite observational hints of supersolar metallicities in the black hole neighbourhood. In fact flatter $\Gamma$ values (Vito et al. 2013) and supersolar metallicities could both significantly reduce the estimated hydrogen column density. Some statistics on the relative fraction of obscured/unobscured QSOs of intermediate X-ray luminosity (43 $\lesssim \log L_{\mathrm{X}} \lesssim 44$ ) show that the fraction of obscured AGNs at high redshifts could be $\sim 50 \%$ (Hasinger 2008; Merloni et al. 2014), although there is no tight correlation between X-ray absorption and the presence of broad emission lines since some X-ray absorbed AGNs show broad emission lines in their restframe UV spectra, as outlined by Fiore et al. (2012). Moreover, recent COS spectral analysis of FUV spectra of low-z AGNs of different luminosities $\left(\log L_{\mathrm{UV}}=44-46.5\right)$ and spectral types (Sy 1, Sy 1.2, Sy 1.5, QSOs) by Shull et al. (2012) and Stevans et al. (2014) show no continuum edges at $912 \AA$. In this complex situation we assume that the presence of any AGN activity in high-z, rest-frame UV selected galaxies - activity certified by $\mathrm{X}$-ray emission at levels $\log L_{\mathrm{X}} \gtrsim 43-$ is able to provide the needed mechanical and radiative feedback mechanisms to allow ionizing UV photons to escape outside the host galaxies into the surrounding IGM with average escape fractions in the sample not much smaller than 1 (in the range $\langle f\rangle \sim 0.7-1$ ). We note that a reduction of the expected photoionization rate by e.g. $30 \%$ with respect to that shown in Fig. 6, due to an assumed average escape fraction $\langle f\rangle \sim 0.7$, would be compensated by the re-emission of ionizing photons by the IGM due to radiative recombination (Haardt \& Madau 1996) that in a framework of reionization by the AGN population could contribute by a factor $\sim 1.25$ to the cosmological photoionization rate at $z \gtrsim 5$ (Haardt, priv. comm.). 
Feedback at various intensity levels is indeed emerging from the most recent observations as a ubiquitous activity in AGNs, independent of their spectral type (e.g. Cicone et al. 2014 and references therein). Because of the feedback mechanisms in the AGN host galaxies some escaping ionizing photons could also come from young stars in the hosts. For a given AGN/stellar flux ratio at $1500 \AA$, the stellar ionizing spectrum at $\lambda \lesssim 912 \AA$ is expected to be softer than shown by the AGN because of intrinsic Lyman continuum absorption in the stellar atmospheres. The latter absorption is essentially unknown and expected values depend on the spectral synthesis models adopted, on the age and metallicity assumed for the stellar populations in star forming galaxies. Typical values for the $F_{1500} / F_{912}$ flux ratio, which is a measure of the intrinsic stellar absorption, are $\sim 3$ for normal star forming galaxies. However, for our AGN host galaxies at $z>4$ the age and metallicity of the associated starburst activity could be $\lesssim 10^{7}$ yr and $\sim 0.2 Z_{\odot}$, respectively, implying intrinsic $F_{1500} / F_{912}$ ratios of $\sim 2$ or smaller. These values are not far from the ratio 1.7 derived from our adopted AGN spectral shape. In other words, the ionizing UV spectra from a very young population in AGN host galaxies could be almost as hard as that of the AGNs at wavelengths just shortward of the Lyman edge at $912 \AA$. Of course with decreasing wavelengths shortward of $912 \AA$ the AGN and stellar spectral shapes are progressively more different and at the HeII edge (228 $)$ ) only the AGN SEDs can contribute to the HeII reionization.

\subsection{Hell reionization}

For this reason, in our proposed scenario for the cosmic reionization, the hardness of the ionizing UV spectrum provided by the AGN population would produce an early significant reionization of the HeII content in the IGM. There is some consensus that HeII reionization could be completed later in the Universe than hydrogen, at $z \sim 3$, supporting a scenario where galaxies with much softer UV spectra dominate the ionization of hydrogen at very high redshifts until AGNs become so numerous at $z \sim 3$ that they provide the required hard photons to reionize HeII. The issue has already been discussed in Giallongo et al. (2012) in the framework of an AGN dominated UV background where the HeIII volume filling factor was computed as a function of decreasing redshift under various assumptions. In particular it was recognized that the presence of optically thick HeII clouds between AGNs reduce the mean free path of HeII ionizing photons, according to Bolton et al. (2009), allowing a gradual increase of the HeII reionization as the redshift decreases up to a full reionization at $z \sim 3$. A detailed evolutionary history of the hydrogen and helium reionization requires 3D hydrodynamical models; however, the simplified model suggests that a modest degree of inhomogeneity in the HeII distribution could delay its reionization history. In this context, recent direct observations of HeII spectral regions in 17 bright QSOs at $z \sim 3$ with HST/COS by Worseck et al. (2014b) seem to suggest a mild evolution of the HeII reionization with increasing redshift allowing the possibility of getting the bulk of the HeII reionization in the Universe at $z>4$. On the other hand, recent detailed hydrodynamical simulations of the IGM (Compostella et al. 2014; Puchwein et al. 2014) show the need for an increase in the hydrogen and HeII photoionization rates with respect to what is predicted by the Haardt \& Madau (2012) model at very high redshifts. If confirmed, these analyses could support a reionization scenario driven by AGNs.

\section{Summary}

Thanks to the completion of deep multiwavelength surveys it is now possible to apply specific selection procedures to reveal the very high redshift AGN population with intrinsic luminosities more typical of local Seyfert galaxies rather than bright Sloan QSOs.

More specifically, we have selected AGN candidates at $z>4$ in the CANDELS GOODS-S field starting from the NIR $H$ band selection of the galaxy parent sample, which corresponds to UV rest-frame selection, down to a very faint magnitude limit $H \leq$ 27. We have used photometric and a few spectroscopic redshifts derived from the CANDELS catalogue and we have extracted from these high- $z$ galaxies the subsample of AGN candidates showing X-ray detection above $F_{\mathrm{X}} \sim 1.5 \times 10^{-17} \mathrm{erg} \mathrm{cm}^{-2} \mathrm{~s}^{-1}$ $(0.5-2 \mathrm{keV})$, corresponding to a probability of spurious detection of $2 \times 10^{-4}$ in the Chandra 4 Ms GOODS-S field.

We have made a preliminary estimate of the selection function aimed at deriving a correction for the sample incompleteness. We have derived average volume densities $10^{-5} \lesssim \phi \lesssim$ $10^{-4} \mathrm{Mpc}^{-3} \mathrm{mag}^{-1}$ in the magnitude interval $-18.5 \gtrsim M_{1450} \gtrsim$ -22.5 . Thus, the presence of even a few faint high- $z$ AGN candidates in the GOODS-S field increases appreciably the cosmological significance of the AGN population at $z=4-6.5$, especially in the context of the cosmic reionization.

To make a first estimate of the ionizing UV emissivity of the AGN population at these high- $z$ we have derived for the first time the shape of the UV luminosity function from $M_{1450} \sim-18.5$ to $M_{1450} \sim-28$ adding the bright QSO samples from the Sloan survey which is less affected by significant incompleteness. The UV luminosity function is well represented by a double power law although the position of the break is highly uncertain. The values obtained from a simple fit are used to derive an ionizing emissivity of the order of $\epsilon_{24} \sim 6.5$ at $z=4-5$ and $\epsilon_{24} \sim 2.5$ at $z \sim 6$, assuming an average AGN UV spectral shape and an escape fraction of ionizing UV photons $\langle f\rangle=1$.

The derived emissivity has then been used to estimate the hydrogen photoionization rate in the same redshift interval which is of the order of $\Gamma_{-12} \sim 0.1$ at $z \sim 6$, consistent with the value required to keep highly ionized the intergalactic medium observed in the Lyman- $\alpha$ forest of bright QSO spectra at the same redshift. This scenario points toward a late reionization epoch at redshifts not much higher than $z=7$ as suggested by the rapid decrease in the number of Lyman- $\alpha$ emitters and by the recent lower Thompson optical depth suggested by recent preliminary Planck data ${ }^{1}$.

We have discussed some caveats which could reduce the volume density and/or ionizing emissivity of the high redshift AGN population described in the present work. More specifically, the large uncertainties involved in photometric redshifts for sources with steep, almost featureless, spectral energy distribution could spuriously enhance the total number of $z>5$ AGN candidates in the GOODS-S field. However, after careful evaluation of this bias, we have shown that possible changes in the AGN volume densities provide values still consistent with the adopted shape of the UV luminosity function in the redshift interval $5<z<6.5$. A further important caveat is related to the escape fraction of ionizing photons from the AGN host galaxies which should be on average $\langle f\rangle \gtrsim 0.5$ to ensure enough ionizing photons in the Universe. At present it is not clear if fainter AGNs ionize their neighbourhood as efficiently as the brighter QSO population does.

http://www . cosmos. esa. int/web/planck/ferrara2014 
We finally emphasize that, given the large uncertainty in the present data, the aim of this preliminary study is not focused on deriving a reliable shape of the AGN UV luminosity function, but rather is devoted to establishing whether faint AGNs can be definitely ruled out as main contributors to the cosmic reionization, as generally thought. The main outcome of the present preliminary analysis goes in the opposite direction. Given the large uncertainties involved, there is room for a significant contribution to reionization by the $z>4$ AGN population under reasonable physical assumptions. Looking for faint NIR selected high$z$ galaxies with even marginal X-ray detection in the deepest available NIR and X-ray images can be a promising technique to reveal a large number of Lyman continuum emitting sources. Applying the same selection strategy and spectroscopic follow up to other fields with similar characteristics such as GOODS-N and COSMOS will provide a larger composite sample and consequently a more reliable estimate of the AGN UV luminosity function and photoionization rates up to redshift $z \gtrsim 6$ where the cosmic hydrogen reionization is still in action.

Acknowledgements. We acknowledge useful comments by the referee that have improved the analysis in the discussion section. We thank F. Haardt for discussion and for giving us preliminary estimates of the IGM ionizing contribution at high redshifts. We thank V. Khaire for noticing a typo in an earlier version of Table 3. We acknowledge financial contribution from the agreement ASIINAF I/009/10/0. This work is based on observations taken by the CANDELS Multi-Cycle Treasury Program with the NASA/ESA HST, which is operated by the Association of Universities for Research in Astronomy, Inc., under NASA contract NAS5-26555. Observations were also carried out using the Very Large Telescope at the ESO Paranal Observatory under Programme IDs LP186.A-0898, LP181.A-0717, LP168.A-0485, ID 170.A-0788, ID 181.A0485, ID 283.A-5052 and the ESO Science Archive under Programme IDs 60.A9284, 67.A-0249, 71.A-0584, 73.A-0564, 68.A-0563, 69.A-0539, 70.A-0048, 64.O-0643, 66.A-0572, 68.A-0544, 164.O-0561, 163.N-0210, 85.A-0961 and 60.A-9120. This work is based in part on observations made with the Spitzer Space Telescope, which is operated by the Jet Propulsion Laboratory, California Institute of Technology under a contract with NASA. Support for this work was provided by NASA through an award issued by JPL/Caltech. A.F. acknowledges the contribution of the EC FP7 SPACE project ASTRODEEP (Ref.No: 312725).

\section{References}

Ashby, M. L. N., Willner, S. P., Fazio, G. G., et al. 2013, ApJ, 769, 80 Avni, Y., \& Bahcall, J. N. 1980, ApJ, 235, 694

Balestra, I, Mainieri, V., Popesso, P., et al. 2010, A\&A, 512, A12

Becker, G. D., \& Bolton, J. S. 2013, MNRAS, 436, 1023

Bolton, J. S., Haehnelt, M. G., Viel, M., \& Springel, V. 2005, MNRAS, 357, 1178

Bolton, J. S., Oh, S. P., \& Furlanetto, S. R. 2009, MNRAS, 395, 736

Boutsia, K., Grazian, A., Giallongo, E., et al. 2011, ApJ, 736, 41

Calverley, A. P., Becker, G. D., Haehnelt, M. G., \& Bolton, J. S. 2011, MNRAS, 412,2543

Cavaliere, A., \& Vittorini, V. 2000, ApJ, 543, 599

Cavaliere, A., Lapi, A., \& Menci, N. 2002, ApJ, 581, L1

Cicone, C., Maiolino, R., Sturm, E., et al. 2014, A\&A, 562, A21

Civano, F. Brusa, M., Comastri, A., et al. 2011, ApJ, 741, 91

Compostella, M., Cantalupo, S., \& Porciani, C. 2014, MNRAS, 445, 4186

Dahlen, T., Mobasher, B., Faber, S. M., et al. 2013, ApJ, 775, 93

Fan X., Strauss, M. A., Becker, R. H., et al. 2006, ApJ, 132, 117

Faucher-Giguere, C.-A., Linz, A., Hernquist, L., \& Zaldarriaga, M. 2008, ApJ, 688,85

Ferrara, A., \& Loeb, A. 2013, MNRAS, 431, 2826

Finkelstein, S. L., Papovich, C., Ryan, R. E., et al. 2012, ApJ, 758, 93
Finkelstein, S. L., Ryan, R. E., Papovich, C., et al. 2014, ApJ, submitted, [arXiv: 1410.5439$]$

Fiore, F., Puccetti, S., Grazian, A., et al. 2012, A\&A, 537, A16

Fontanot, F., Cristiani, S., Pfrommer, C., Cupani, G., \& Vanzella, E. 2014, MNRAS, 438, 2097

Gehrels, N. 1986, ApJ, 303, 336

George, E. M., Reichardt, C. L., Aird, K. A., et al. 2015, ApJ, 799, 177

Giallongo, E., Menci, N., Fiore, F., et al. 2012, ApJ, 755, 124

Gilli, R., Norman, C., Vignali, C., et al. 2014, A\&A, 562, A67

Glikman, E., Djorgovski, S. G., Stern, D., et al. 2011, ApJ, 728, L26

Grazian, A., Fontana, A., Santini, P., et al. 2015, A\&A, 575, A96

Grogin, N. A., Kocevski, D. D., Faber, S. M., et al. 2011, ApJS, 197, 35

Guo, Y., Ferguson, H. C., Giavalisco, M., et al. 2013, ApJS, 207, 24

Haardt, F., \& Madau, P. 1996, ApJ, 461, 20

Haardt, F., \& Madau, P. 2012, ApJ, 746, 125

Hasinger, G. 2008, A\&A, 490, 905

Hathi, N. P., Malhotra, S., \& Rhoads, J. E. 2008, ApJ, 673, 686

Hinshaw, G., Larson, D., Komatsu, E., et al. 2013, ApJS, 208, 19

Hsu, L., Salvato, M., Nandra, K., et al. 2015, ApJ, 796, 60

Ishigaki, M., Kawamata, R., Ouchi, M., et al. 2015, ApJ, 799, 12

Kashikawa, N., Ishizaki, Y., Willott, C. J., et al. 2015, ApJ, 798, 28

Kirkman, D., Tytler, D., Suzuki, N., et al. 2005, MNRAS, 360, 1373

Koekemoer, A. M., Faber, S. M., Ferguson, H. C., et al. 2011, ApJS, 197, 36

Lapi, A., Cavaliere, A., \& Menci, N. 2005, ApJ, 619, 60

Luo, B., Bauer, F. E., Brandt, W. N., et al. 2008, ApJS, 179, 19

Masters, D., Capak, P., Salvato, M., et al. 2012, ApJ, 755, 169

Menci, N., Fontana, A., Giallongo, E., \& Salimbeni, S. 2005, ApJ, 632, 49

Menci, N., Fiore, F., Puccetti, S., \& Cavaliere, A. 2008, ApJ, 686, 219

Menci, N., Fiore, F., \& Lamastra, A. 2013, ApJ, 766, 110

Merloni, A., Bongiorno, A., Brusa, M., et al. 2014, MNRAS, 437, 3550

Miralda-Escudé, J., Haehnelt, M., \& Rees, M. J. 2000, ApJ, 530, 1

Mostardi, R. E., Shapley, A. E., Nestor, D. B., et al. 2013, ApJ, 779, 65

Nestor, D. B., Shapley, A. E., Steidel, C. C., \& Siana, B. 2011, ApJ, 736, 18

Pentericci, L., Fontana, A., Vanzella, E., et al. 2011, ApJ, 743, 132

Pentericci, L., Vanzella, E., Fontana, A., et al. 2014, ApJ, 793, 113

Prochaska, J. X., Worseck, G., \& O’Meara, J. M. 2009, ApJ, 705, L113

Puchwein, E., Bolton, J. S., Haehnelt, M. G., Madau, P., \& Becker, G. D. 2014 MNRAS, submitted [arXiv: 1410.1531]

Rhoads, J. E., Malhotra, S., Pirzkal, N., et al. 2009, ApJ, 697, 942

Robertson, B. E., Furlanetto, S. R., Schneider, E., et al. 2013, ApJ, 768, 71

Salimbeni, S., Giallongo, E., Menci, N., et al. 2008, A\&A, 477, 763

Sargent, W. L. W., Steidel, C. C., \& Boksenberg, A. 1989, ApJS, 69, 703

Schirber, M., \& Bullock, J. S. 2003, ApJ, 584, 110

Schmidt, M. 1968, ApJ, 151, 393

Shaerer, D., \& de Barros, S. 2009, A\&A, 502, 423

Shankar, F., \& Mathur, S. 2007, ApJ, 660, 1051

Shull, J. M., Stevans, M. L., \& Danforth, C. W. 2012, ApJ, 752, 162

Songaila, A., \& Cowie, L. L. 2010, ApJ, 721, 1448

Stevans, M. L., Shull, J. M., Danforth, C. W., \& Tilton, E. M. 2014, ApJ, 794, 75

Telfer, R. C., Zheng, W. K., Gerard, A., \& Davidsen, A. F. 2002, ApJ, 565, 773

Tilvi, V., Papovich, C., Finkelstein, S. L., et al. 2014, ApJ, 794, 5

Treu, T., Schmidt, K. B., Trenti, M., Bradley, L. D., \& Stiavelli, M. 2013, ApJ, 775,29

Vanden Berk, D. E., Richards, G. T., Bauer, A., et al. 2001, AJ, 122, 549

Vanzella, E., Cristiani, S., Dickinson, M., et al. 2008, A\&A, 478, 83

Vanzella, E., Siana, B., Cristiani, S., \& Nonino, M. 2010, MNRAS, 404, 1672

Vanzella, E., Guo, Y., Giavalisco, M., et al. 2012a, ApJ, 751, 70

Vanzella, E., Nonino, M., Cristiani, S., et al. 2012b, MNRAS, 424, 54

Vito, F., Vignali, C., Gilli, R., et al. 2013, MNRAS, 428, 354

Vito, F., Gilli, R., Vignali, C., et al. 2014, MNRAS, 445, 3557

Volonteri, M. 2012, Science, 337, 544

Volonteri, M., \& Silk, J. 2014, ApJ, 804, 148

Weigel, A. K., Schawinski, K., Treister, E., et al. 2015, MNRAS, 448, 3167

Wyithe, J. S. B., \& Bolton, J. S. 2011, MNRAS, 412, 1926

Wise, J. H., Demchenko, V. G., Halicek, M. T., et al. 2014, MNRAS, 442, 2560

Worseck, G., Prochaska, J. X., O’Meara, J. M., et al. 2014a, MNRAS, 445, 1745

Worseck, G., Prochaska, J. X., Hennawi, J. F., \& McQuinn, M. 2014b, ApJ, submitted [arXiv: 1405.7405]

Xue, Y. Q., Luo, B., Brandt, W. N., et al. 2011, ApJS, 195, 10

Yue, B., Ferrara, A., Vanzella, E., \& Salvaterra, R. 2014, MNRAS, 443, L20

Pages 13 to 14 are available in the electronic edition of the journal at http://www . aanda. org 


\section{Appendix A: Spectral energy distributions and redshift probability distribution functions}

In this section we show the spectral energy distributions (SEDs) and the probability distribution functions (PDFs) of the photometric redshift estimates for each AGN candidate derived from the Dahlen el al. (2013) analysis. Most of the candidates in Fig. A.1 show PDFs confined at $z>4$ with only small wings at $z<4$. The most uncertain redshifts are for the objects 20765 , 28476,29323 , and 33160 , although the larger PDFs distributions are mostly distributed at $z>4$. We note that the last three objects at $z>6$ are among the most uncertain owing to the combination of the power-law shape of their SEDs and the faintness of the sources. We also note that for some of these objects, the Bayesian photometric redshift does not correspond to the peak of the PDF, but is the result of a weighted average around the maximum.
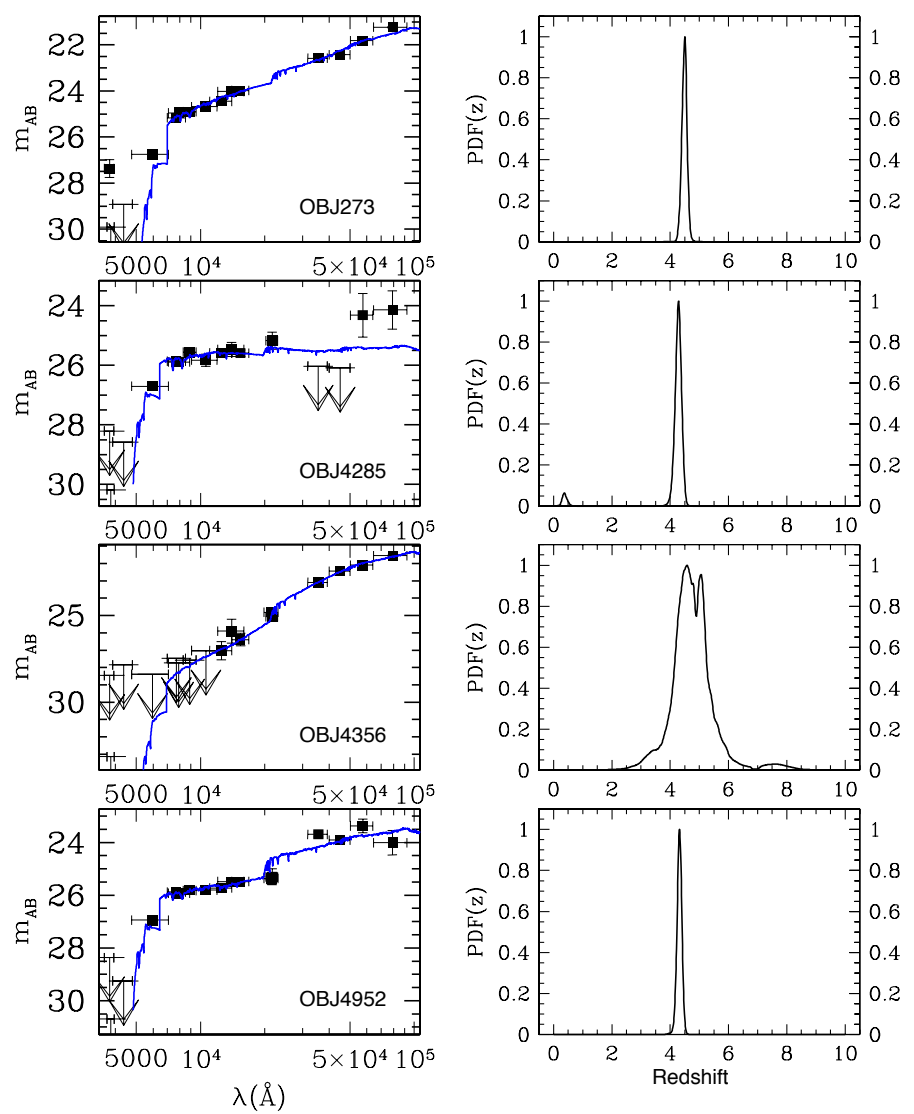

Fig. A.1. Spectral energy distributions and probability distribution functions of photometric redshifts normalized to 1 at the peak for all the AGN candidates shown in Table 2. $1 \sigma$ upper limits are shown as downward arrows.
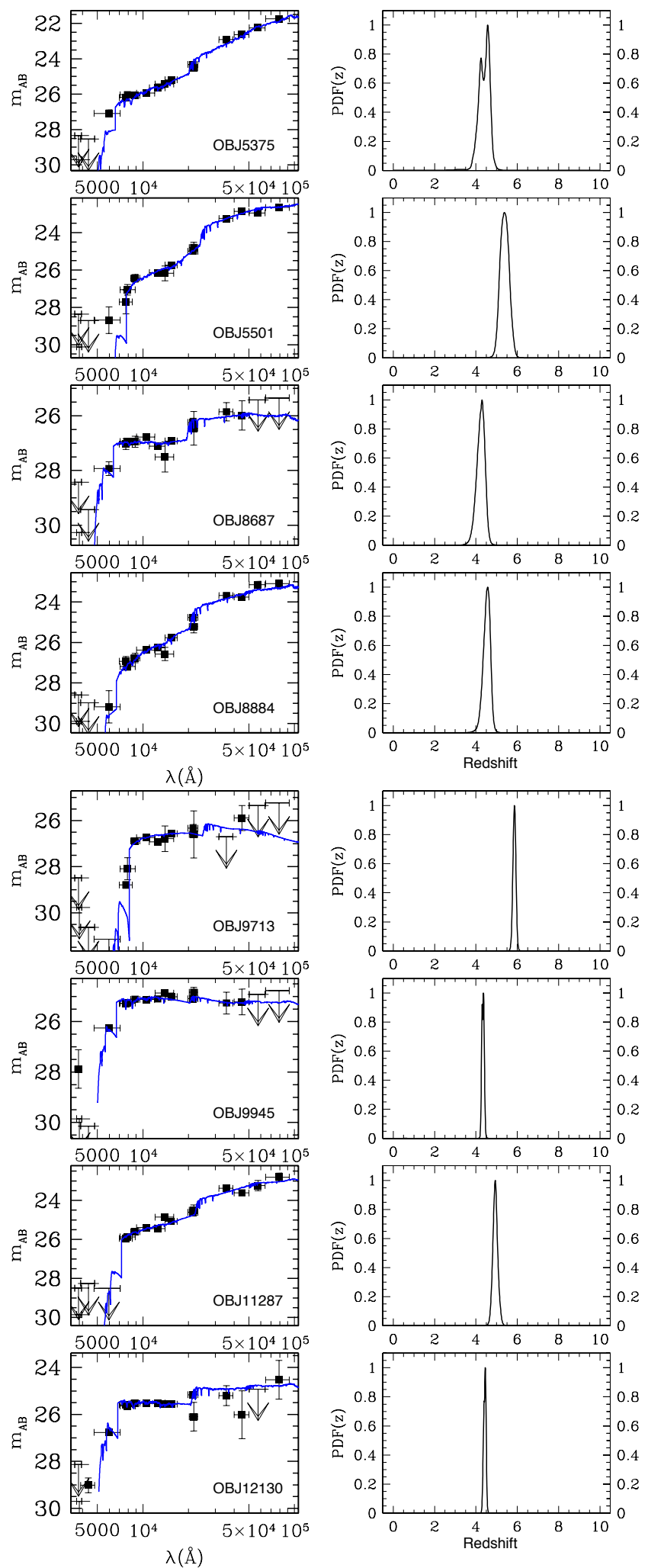

Fig. A.1. continued. 

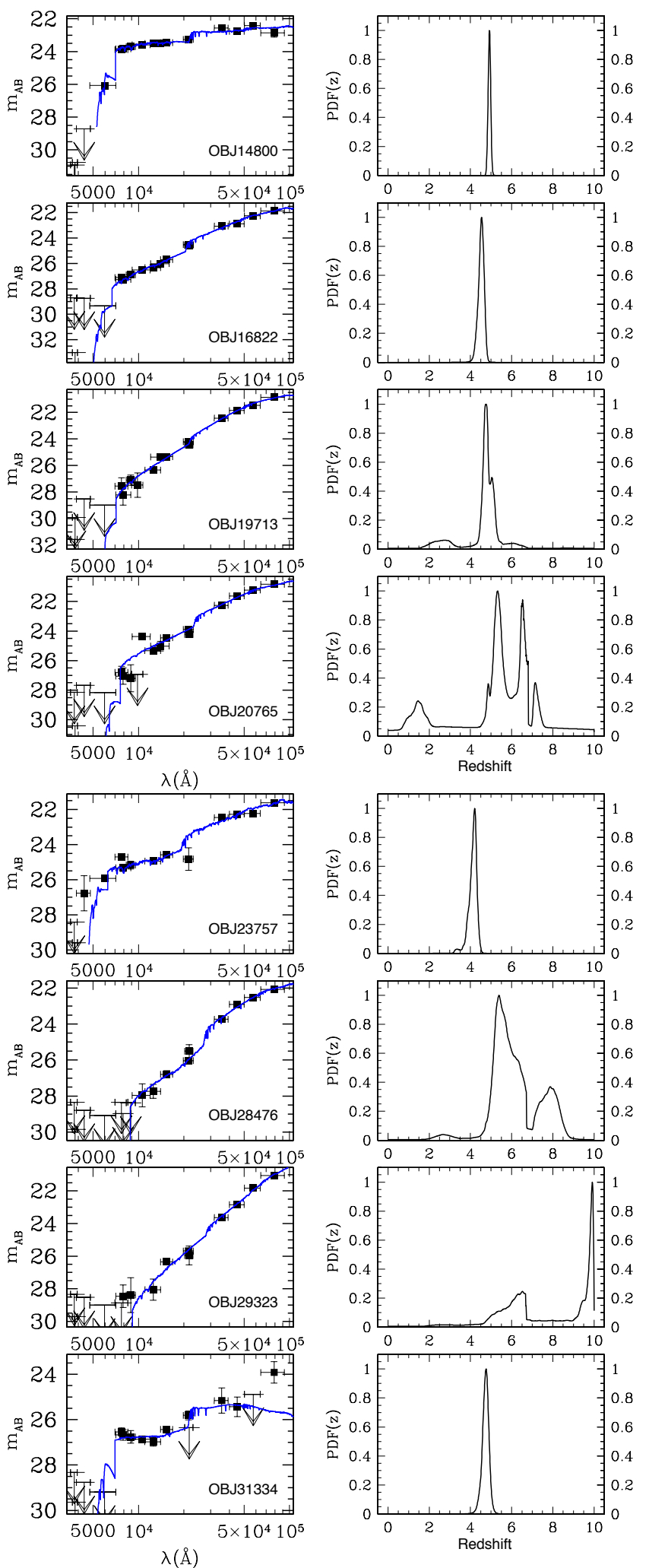

Fig. A.1. continued.
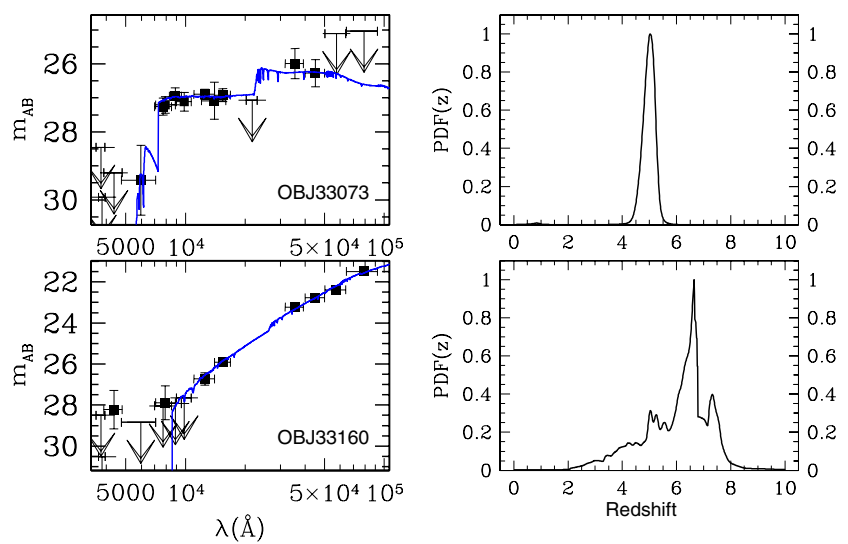

Fig. A.1. continued.

\section{Appendix B: X-ray position accuracy}

In this section we show an example of the relative position accuracy between the X-ray and $H$ band image. We have selected the object 28476 already shown in Fig. 1 which is located near two brighter $H$ band sources. In Fig. B.1 we also show the position accuracy of a bright source in the bottom left corner, comparable to that of 28476. In general the position accuracy between the $H$ and X-ray sources is $\lesssim 1$ arcsec for all the AGN candidates in our sample. They have off-axis Chandra positions $<9$ arcmin.

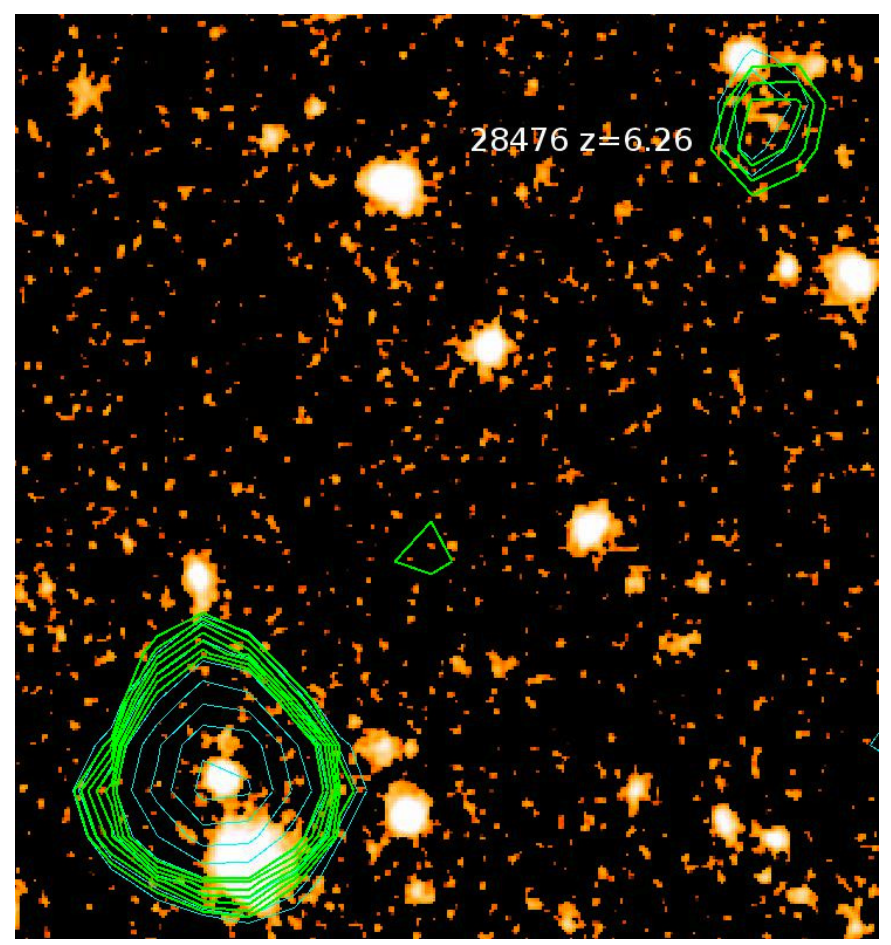

Fig. B.1. $H$ band image around the AGN candidate 28476 (upper right) shown in Fig. 1. The size is 30 arcsec. Cyan and green contours are X-ray detections in the $0.8-4 \mathrm{keV}$ and $0.5-2 \mathrm{keV}$ bands, respectively. Position accuracies are within 1 arcsec. 